Models of spin-orbit coupled oligomers

\title{
Models of spin-orbit-coupled oligomers
}

G. Gligorić, ${ }^{1}$ A. Radosavljević, ${ }^{1}$ J. Petrović, ${ }^{1}$ A. Maluckov, ${ }^{1}$ Lj. Hadžievski, ${ }^{1}$ and B. A. Malomed $^{2}$

1) $P^{*}$ group, Vinča Institute of Nuclear Sciences, University of Belgrade, P. O. B. 522, 11001 Belgrade, Serbia

2) Department of Physical Electronics, School of Electrical Engineering, Faculty of Engineering, and Center for Light-Matter Interaction, Tel Aviv University, Tel Aviv 69978, Israel ITMO University, St. Petersburg 197101, Russia

(Dated: 22 June 2021)

We address the stability and dynamics of eigenmodes in linearly-shaped strings (dimers, trimers, tetramers, and pentamers) built of droplets of a binary BoseEinstein condensate (BEC). The binary BEC is composed of atoms in two pseudo-spin states with attractive interactions, dressed by properly arranged laser fields, which induce the (pseudo-) spin-orbit (SO) coupling. We demonstrate that the SO-coupling terms help to create eigenmodes of particular types in the strings. Dimer, trimer, and pentamer eigenmodes of the linear system, which correspond to the zero eigenvalue (EV, alias chemical potential) extend into the nonlinear ones, keeping an exact analytical form, while tetramers do not admit such a continuation, because the respective spectrum does not contain a zero EV. Stability areas of these modes shrink with the increasing nonlinearity. Besides these modes, other types of nonlinear states, which are produced by the continuation of their linear counterparts corresponding to some nonzero EVs, are found in a numerical form (including ones for the tetramer system). They are stable in nearly entire existence regions in trimer and pentamer systems, but only in a very small area for the tetramers. Similar results are also obtained, but not displayed in detail, for hexa- and septamers.

PACS numbers: 42.82.Et; 03.65.Ge; 03.75.Mn 
Discrete (lattice) dynamical systems, built with intersite linear coupling and onsite nonlinearity, are a subject of theoretical and experimental studies in a vast research area. While the dynamics of large-size dynamical lattices may be quite complex, in many cases insight into their fundamental dynamical properties is provided by truncation to small networks. In the simplest case, these are onedimensional strings including several lattice sites. Recently, a new paradigm of combined linear and nonlinear dynamics was introduced in two-component BoseEinstein condensates (BECs), with the components which are linearly mixed, through the first-order spatial derivatives, by the (pseudo-) spin-orbit (SO) coupling. It has been found that the interplay of linear and nonlinear interactions, specific to the SO-coupled BEC, gives rise to many unusual modes. In particular, two-dimensional solitons in the free space, which are always unstable in conventional systems, may be stabilized by the SO coupling. This analysis produces novel results in continuous and discrete versions of the nonlinear SO-coupled system alike. The objective of this work is to identify stable nonlinear modes in finite strings, composed of two, three, four, or five lattice sites (oligomers), under the combined action of the onsite cubic self- and cross-attraction and linear SO coupling, in the two-component BEC. In the dynamical strings considered here, new modes are found. These include, on the one hand, linear eigenmodes corresponding to zero eigenvalues (EVs), which can be extended without any change in shape in the presence of nonlinearity, and, on the other hand, nonlinear states which, in the trimer and pentamer systems, originate from eigenmodes associated with some nonzero eigenvalues in the limit of vanishing nonlinearity, but have their shapes different from those of the linear counterparts. In the tetramer system, a family of nonlinear modes is found too, but, on the contrary to those in the trimer and pentamer cases, its stability range is very narrow. Lastly, the generality of the findings is confirmed by the fact that similar results are obtained (but not reported here in detail) for the oligomers built of six and seven sites. 
Models of spin-orbit coupled oligomers

\section{INTRODUCTION}

The behavior of complex networks with interactions of various types between constituents is a vast research area with diverse applications to physics and other fields of science ${ }^{1,2}$. To address a more particular and practically tractable class of such systems, we here study small sets (strings) formed of $N$ coupled pseudospinor (two-component) droplets of atomic Bose-Einstein condensates (BECs), which is an important physical setting ${ }^{3}$, that can be realized in the experiment by means of deep optical-lattice potentials $\underline{4}, \underline{5}$. The corresponding eigenmodes were found, and effects of the linear spin-orbit (SO) coupling ${ }^{6}-\underline{9}$ and nonlinear intra- and inter- component interactions on their shape and stability were studied in detail.

The background of our analysis refers to light transmission in networks consisting of a small number $(N)$ of linearly coupled fibers, or waveguides mounted on a chip. These systems were analytically studied in special cases, such as dimers, various forms of trimer configurations $\frac{10}{-17}$ some other very specific settings $\frac{18}{}$. In addition, the self-trapping transition in dimers was studied analytically and numerically, with respect to the occurrence of a bifurcation in the stationary states of the system $\frac{19}{}$ and its temporal dynamics 11 . The actual systems are formulated below in terms of BEC, rather than arrayed optical waveguides, as the former interpretation is more straightforward and realistic $20,21$.

The 1D SO-coupled $N$-mer BEC systems with $2 N$ degrees of freedom, built as strings of $N=2,3,4,5$ adjacent BEC droplets, can be defined as a straightforward truncation of the discrete lattice for fields $\phi_{n}^{+,-}$, introduced in Ref. ${ }^{22}$, with the discrete coordinate taking values $n=0,1(N=2), n=0, \pm 1(N=3), n=0, \pm 1,2(N=4)$, and $n=0, \pm 1, \pm 2(N=5)$, respectively. Accordingly, the evolution of the wave function is governed by the system of $2 N$ coupled discrete Gross-Pitaevskii equations (GPEs). The peculiarity of these systems is the double number of degrees of freedom due to the presence of two coupled components of the underlying spinor wave function. Moreover, the coupling constants (parameters) may take complex values, which contributes to diversity of the eigenmodes in these small systems, although the structure of some modes is simplified by specific symmetries.

In contrast to convoluted situations in solid-state physics, the "synthetic" SO coupling, induced by the appropriate laser illumination of atomic gases in the combination with a magnetic field, can be precisely controlled in the experiment $\underline{\underline{6}-\underline{\underline{9}}}$. The link between the solidstate and atomic-gas settings is established by mapping the electrons' spinor wave functions 
onto pseudo-spinor mean-field wave functions of the binary BEC, which is composed of atoms in two different states, "dressed" by the laser fields. In particular, making use of the electronic ground-state hyperfine manifolds $5 S_{1 / 2}, F=1$ in ${ }^{87} \mathrm{Rb}$, one can start with the set of two hyperfine ground states, viz., $\left|F=1, m_{F}=-1\right\rangle$ and $\left|F=1, m_{F}=0\right\rangle$. This pair of atomic states emulate the set of spin-up $(+)$ and spin-down $(-)$ components of the electrons' wave functions. The $1 \mathrm{D}$ discrete model with the intrinsic SO coupling of the Rashba type and attractive nonlinearity, introduced in Refs $\underline{22} \underline{24} \underline{29}$, is adopted in the present work to predict various steady states and their stability in strings of tunnel-coupled BEC droplets. As mentioned above, the possibility to use deep optically induced potentials makes such strings objects of direct relevance to the experiment $\underline{3.4}$.

The rest of the paper is structured as follows. The model equations, their basic properties, and a brief overview of numerical procedures employed in the present work are introduced in Sec. II. In Sections III - V, nonlinear modes in dimer, trimer, tetramer and pentamer systems of coupled droplets are addressed in necessary details. The presentation in each section starts with the analysis of the eigenvalue problem of the corresponding linear system, without and with the SO coupling. The next step is searching for nonlinear eigenmodes which are a direct continuation of linear ones, and the analysis of their stability. Such nonlinear solutions are found, in an exact analytical form, in the dimer, trimer, and pentamer systems, for the modes which correspond to zero chemical potential (eigenfrequency), $\mu$, in the linear limit, but they do not exists in the case of tetramers. Some linear eigenmodes corresponding to $\mu \neq 0$ can

be also be extended to the nonlinear systems, but in a numerical form. Nonlinear modes of the latter type in trimers, tetramers, and pentamers are reported in Section VI. The paper is concluded by Section VII. In particular, in that section we mention that results similar to those for tetramers have been obtained in the hexamer system (another one with an even number of sites), and results similar to what has been found for trimers and pentamers were also produced for septamers (the next system with an odd number), although the latter cases are not reported in the paper, to keep its size in reasonable limits.

\section{THE MODEL EQUATIONS}

The fragmentation of the BEC in the deep optical lattice (OL) potential into droplets, coupled by tunnelling across barriers separating local potential wells, leads to the replace- 
ment of the continuous Gross-Pitaevskii equations (GPEs), which provide for the mean-field approximation, by their discrete counterparts. This can be performed using the tight-binding approximation as it was elaborated in Refs. ${ }^{22,23,25}$ for the SO-coupled pseudo-spinor BEC wave function, $\Phi_{n}=\left(\phi_{n}^{+}, \phi_{n}^{-}\right)^{T}$, where $n$ is the discrete coordinate:

$$
\begin{aligned}
& i \frac{d \phi_{n}^{+}}{d t}=-\frac{1}{2}\left(\phi_{n-1}^{+}+\phi_{n+1}^{+}\right)-\left(\beta\left|\phi_{n}^{+}\right|^{2}+\kappa\left|\phi_{n}^{-}\right|^{2}\right) \phi_{n}^{+}+\lambda\left(-\phi_{n-1}^{-}+\phi_{n+1}^{-}\right), \\
& i \frac{d \phi_{n}^{-}}{d t}=-\frac{1}{2}\left(\phi_{n-1}^{-}+\phi_{n+1}^{-}\right)-\left(\beta\left|\phi_{n}^{-}\right|^{2}+\kappa\left|\phi_{n}^{+}\right|^{2}\right) \phi_{n}^{-}+\lambda\left(\phi_{n-1}^{+}-\phi_{n+1}^{+}\right) .
\end{aligned}
$$

Here, components $\phi_{n}^{ \pm}$represent two different hyperfine atomic states, $\beta>0$ and $\kappa>0$ are strengths of the self- and cross-attraction of the two components, respectively, and $\lambda$ is the SO-coupling strength. The case of the Manakov's nonlinearity, with $\beta=\kappa$, will play a special role in the analysis presented below, as it is close to the real situation in binary BECs, where the intra- and inter-component scattering lengths are almost exactly equal 26 . By means of rescaling, we fix $\beta=1$.

Coupling constant $\lambda$ is taken real and positive in Eq. (1) because, starting from a complex or negative one, $\lambda=|\lambda| \exp (i \chi)$ (then, it appears as $\lambda^{*}$ in the equation for $\phi_{n}^{-}$, with the asterisk standing for the complex conjugate), its phase can be eliminated by means of a simple phase shift of the discrete fields, $\phi_{n}^{ \pm} \rightarrow \phi_{n}^{ \pm} \exp ( \pm i \chi / 2)$. While Eq. (11) assumes the attractive on-site interaction, the originally repulsive sign can be transformed into the attractive one by means of the well-known staggering transformation $\frac{27}{}, \Phi_{n}=(-1)^{n} \Phi_{n}^{*}$.

We here consider the one-dimensional SO-coupled $N$-mer BEC systems with $2 N$ degrees of freedom, built as strings of $N=2,3,4,5$ adjacent droplets. These systems are introduced as a straightforward truncation of the full discrete model given by Eq.(1) for fields $\phi_{n}^{ \pm}$, with the discrete coordinate taking values $n=0,1(N=2), n=0, \pm 1(N=3), n=0, \pm 1,2(N=$ 4) and $n=0, \pm 1, \pm 2(N=5)$, respectively. Accordingly, at $n$ which does not belong to these sets, we set $\phi_{n}^{ \pm} \equiv 0$ (for example, $\phi_{-1}^{ \pm}=\phi_{2}^{ \pm} \equiv 0$ in the case of $N=2$ ).

We have also looked for a special type of solutions which obey constraint

$$
\phi_{n}^{+}= \pm i \phi_{n}^{-}
$$

In that case, system Eq.(11) can be reduced to the following single discrete nonlinear Schrödinger equation (DNLSE):

$$
i \frac{d \phi_{n}}{d t}=-\left(\frac{1}{2}+i \lambda\right) \phi_{n-1}-\left(\frac{1}{2}-i \lambda\right) \phi_{n+1}-(1+\kappa)\left|\phi_{n}\right|^{2} \phi_{n} .
$$


Further, substitution $\phi_{n} \equiv\left(\frac{1+2 i \lambda}{\sqrt{1+4 \lambda^{2}}}\right)^{n} \varphi_{n}$ and $\tau \equiv \sqrt{1+4 \lambda^{2}} t$ transforms Eq. (3) into the standard DNLSE:

$$
i \frac{d \varphi_{n}}{d \tau}=-\frac{1}{2}\left(\varphi_{n-1}+\varphi_{n+1}\right)-\frac{1+\kappa}{\sqrt{1+4 \lambda^{2}}}\left|\varphi_{n}\right|^{2} \varphi_{n}
$$

Solutions of such systems of different sizes $N$ have been studied in detail in previous works 11 .

The stability of various stationary modes found below is checked by means of the linear stability analysis (LSA) and by direct numerical simulations. Briefly speaking, the LSA is performed for linearized (Bogoliubov - de Gennes) equations for small perturbations added to

the eigenmodes 25 , 28 $: \tilde{\phi_{\mathbf{n}}^{ \pm}}=\phi_{\mathbf{n}}^{ \pm}+\delta \phi_{\mathbf{n}}^{ \pm},\left|\delta \phi_{\mathbf{n}}^{ \pm}\right| \ll\left|\phi_{\mathbf{n}}^{ \pm}\right|$, where $\tilde{\phi}_{\mathbf{n}}^{ \pm}$and $\phi_{\mathbf{n}}^{ \pm}$denote, respectively, the perturbed discrete solution, and their unperturbed counterparts. Perturbation eigenmodes are looked for as $\delta \phi_{n}^{ \pm}(t)=\delta \phi_{n}(t=0)^{ \pm} \exp (\rho t)$, where $\rho$ is a complex eigenvalue (EV) whose positive real part, if any, implies the instability of the solution. The instability is categorized as exponential, if the corresponding imaginary part of $\rho$ is zero, or as an oscillatory instability otherwise. The perturbed solution is substituted into the underlying equations, which are linearized with respect to the small perturbations. By means of a straightforward algebraic procedure, the resulting system may be reduced to the EV problem with the corresponding matrix ${ }^{28}$, which is then solved numerically .

To confirm the LSA predictions and fully explore the (in)stability of the nonlinear modes, the evolution of the modes, with small random perturbations of relative amplitude 0.01 added to them, was numerically simulated by means of the six-order Runge-Kutta algorithm. The numerical convergence was checked at each step - in particular, by monitoring the conservation of the norm and Hamiltonian (energy).

\section{THE SPIN-ORBIT-COUPLED DIMER}

The SO-coupled dimer system with four degrees of freedom, built of two adjacent sites, can be introduced as a straightforward truncation of the discrete model for fields $\phi_{n}^{ \pm}$, given by Eq. (1), keeping solely discrete coordinates $n=0$ and +1 :

$$
\begin{aligned}
& i \frac{d \phi_{0}^{+}}{d t}=-\frac{1}{2} \phi_{+1}^{+}-\left(\left|\phi_{0}^{+}\right|^{2}+\kappa\left|\phi_{0}^{-}\right|^{2}\right) \phi_{0}^{+}+\lambda \phi_{+1}^{-}, \\
& i \frac{d \phi_{0}^{-}}{d t}=-\frac{1}{2} \phi_{+1}^{-}-\left(\left|\phi_{0}^{-}\right|^{2}+\kappa\left|\phi_{0}^{+}\right|^{2}\right) \phi_{0}^{-}-\lambda \phi_{+1}^{+},
\end{aligned}
$$


Models of spin-orbit coupled oligomers

$$
\begin{aligned}
& i \frac{d \phi_{+1}^{+}}{d t}=-\frac{1}{2} \phi_{0}^{+}-\left(\left|\phi_{+1}^{+}\right|^{2}+\kappa\left|\phi_{+1}^{-}\right|^{2}\right) \phi_{+1}^{+}-\lambda \phi_{0}^{-}, \\
& i \frac{d \phi_{+1}^{-}}{d t}=-\frac{1}{2} \phi_{0}^{-}-\left(\left|\phi_{+1}^{-}\right|^{2}+\kappa\left|\phi_{+1}^{+}\right|^{2}\right) \phi_{+1}^{-}+\lambda \phi_{0}^{+} .
\end{aligned}
$$

The conserved energy of the dimer can be written as

$$
\begin{gathered}
E_{0 .+1}=-\operatorname{Re}\left[\left(\phi_{0}^{+}\right)^{*} \phi_{+1}^{+}+\left(\phi_{0}^{-}\right)^{*} \phi_{+1}^{-}\right] \\
-\frac{1}{2}\left(\left|\phi_{0}^{+}\right|^{4}+\left|\phi_{+1}^{+}\right|^{4}+\left|\phi_{0}^{-}\right|^{4}+\left|\phi_{+1}^{-}\right|^{4}\right)-\kappa\left(\left|\phi_{0}^{+}\right|^{2}\left|\phi_{0}^{-}\right|^{2}+\left|\phi_{+1}^{+}\right|^{2}\left|\phi_{+1}^{-}\right|^{2}\right) \\
+\lambda\left[\left(\phi_{0}^{+}\right)^{*} \phi_{+1}^{-}-\left(\phi_{+1}^{+}\right)^{*} \phi_{0}^{-}-\left(\phi_{0}^{-}\right)^{*} \phi_{+1}^{+}+\left(\phi_{+1}^{-}\right)^{*} \phi_{0}^{+}\right],
\end{gathered}
$$

and its norm is

$$
P=\sum_{+,-}\left(\left|\phi_{0}^{ \pm}\right|^{2}+\left|\phi_{+1}^{ \pm}\right|^{2}\right)
$$

Stationary solutions of system (5) -(6) with real chemical potential $\mu$ are looked for as

$$
\phi_{0,+1}^{ \pm}=\exp (-i \mu t) u_{0,+1}^{ \pm}
$$

After the substitution of expression (9) in the linear version of Eqs. (5)-(6), the following two double-degenerate EVs are obtained:

$$
\mu_{1,2}^{(0)}=\frac{1}{2} \sqrt{1+4 \lambda^{2}}, \mu_{3,4}^{(0)}=-\frac{1}{2} \sqrt{1+4 \lambda^{2}},
$$

where the upper index (0) denotes that the linear system is considered.

The corresponding eigenvectors, displayed in Fig. 1, are

$$
\begin{aligned}
& \mathbf{u}_{1}=\left\{u_{0}^{+}, u_{1}^{+}, u_{0}^{-}, u_{1}^{-}\right\}=C\left\{\frac{-2 \lambda}{\sqrt{1+4 \lambda^{2}}}, 0, \frac{1}{\sqrt{1+4 \lambda^{2}}}, 1\right\}, \\
& \mathbf{u}_{\mathbf{2}}=\left\{u_{0}^{+}, u_{1}^{+}, u_{0}^{-}, u_{1}^{-}\right\}=C\left\{\frac{1}{\sqrt{1+4 \lambda^{2}}}, 1, \frac{2 \lambda}{\sqrt{1+4 \lambda^{2}}}, 0\right\}, \\
& \mathbf{u}_{\mathbf{3}}=\left\{u_{0}^{+}, u_{1}^{+}, u_{0}^{-}, u_{1}^{-}\right\}=C\left\{\frac{2 \lambda}{\sqrt{1+4 \lambda^{2}}}, 0, \frac{-1}{\sqrt{1+4 \lambda^{2}}}, 1\right\} . \\
& \mathbf{u}_{4}=\left\{u_{0}^{+}, u_{1}^{+}, u_{0}^{-}, u_{1}^{-}\right\}=C\left\{\frac{-1}{\sqrt{1+4 \lambda^{2}}}, 1, \frac{-2 \lambda}{\sqrt{1+4 \lambda^{2}}}, 0\right\},
\end{aligned}
$$

where $C$ is an arbitrary constant. In the absence of the SO coupling, $\lambda=0$, the plus and minus components are decoupled, and the system can be reduced to the single-component dimer.

Linear eigenvectors $\left(\mathbf{u}_{\mathbf{1}}, \mathbf{u}_{\mathbf{2}}, \mathbf{u}_{\mathbf{3}}, \mathbf{u}_{\mathbf{4}}\right)$, given by Eq. (11), keep their form (shown in Fig. 1) in the presence of the nonlinearity, but solely in the above-mentioned Manakov's case, when 
Models of spin-orbit coupled oligomers

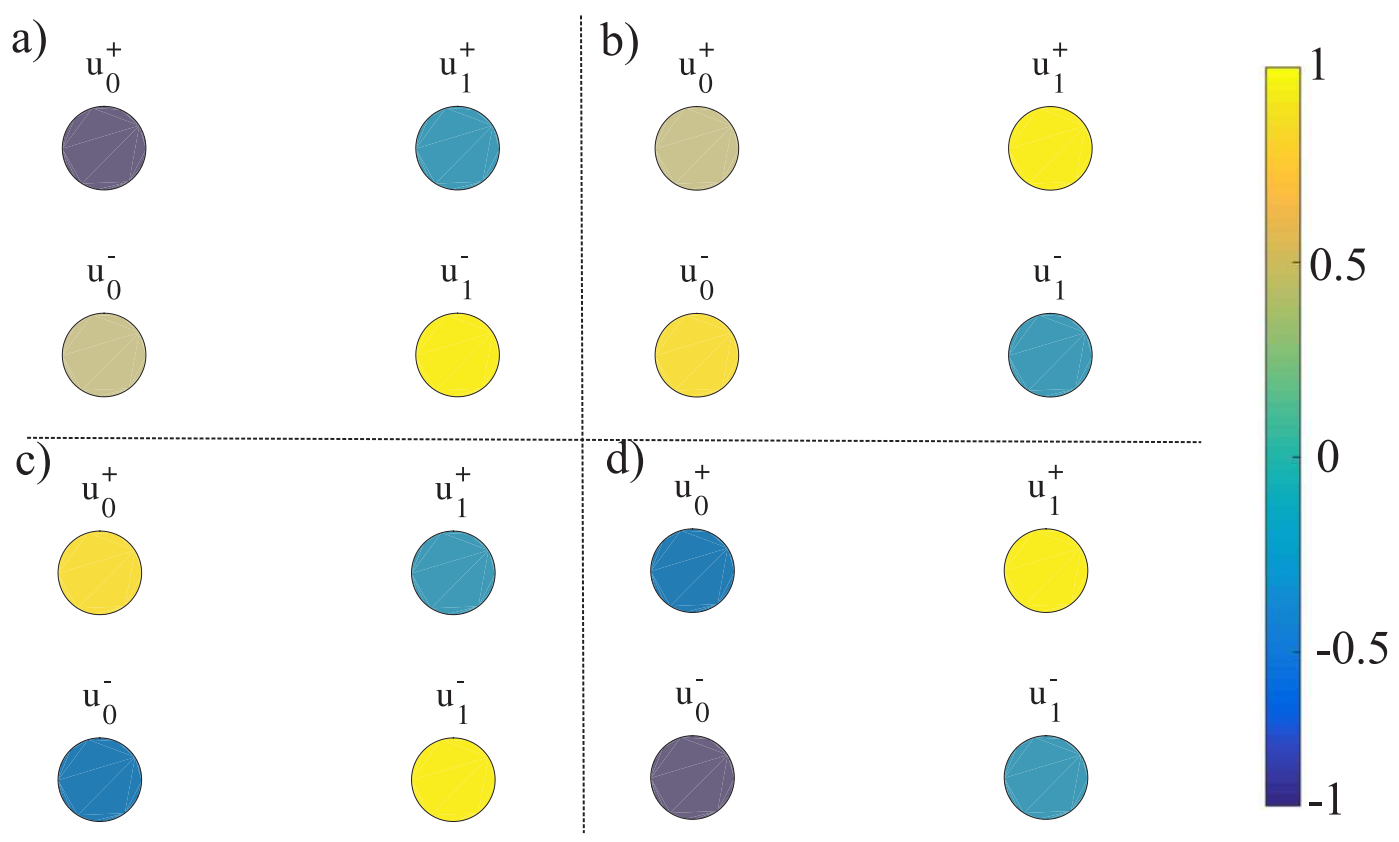

FIG. 1. Four eigenvectors of the linear dimer system that correspond to $\mu_{1,2}^{(0)}(\mathrm{a}, \mathrm{b})$ and $\mu_{3,4}^{(0)}(\mathrm{c}, \mathrm{d})$, given by Eq. (10), at $\lambda=1$.

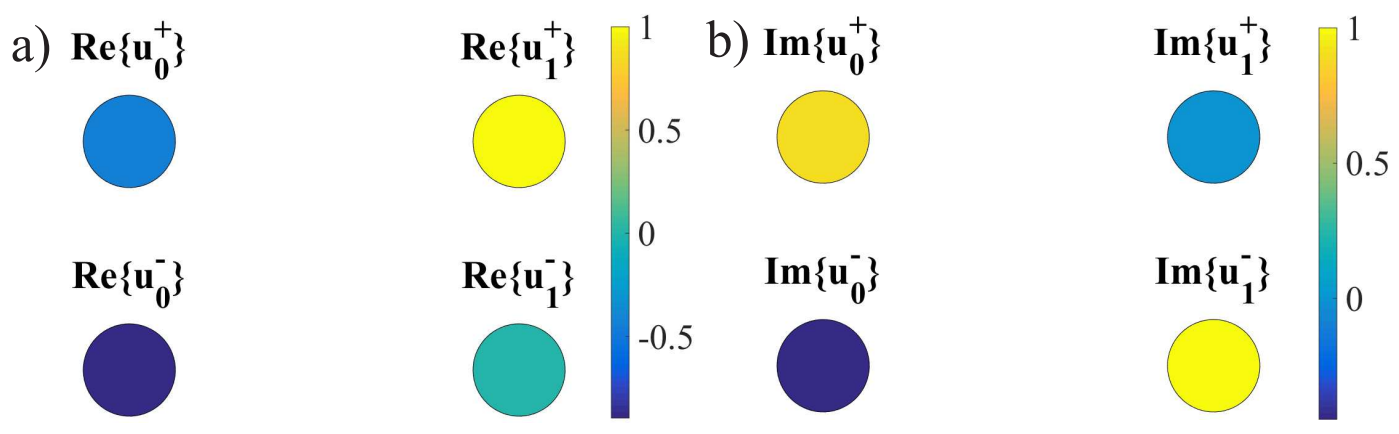

FIG. 2. The real and imaginary parts of the combinations of eigenvector $\mathbf{u}_{\mathbf{4}}+i \mathbf{u}_{\mathbf{3}}$ [see Eq. (16)], which provide solutions for the nonlinear dimer system at $\kappa=1.5$ and $\lambda=1$.

the intra- and inter-component nonlinearity coefficients are equal, $\kappa=1$. This fact may be considered as a manifestation of the principle that extension of linear modes into nonlinear ones is facilitated if nonlinear terms in the system obey the same symmetry as linear ones, see, e.g. Ref. $\underline{\underline{29}}$. The corresponding EVs of the chemical potential, including the nonlinear shift, are

$$
\mu=-|C|^{2} \pm \frac{1}{2} \sqrt{1+4 \lambda^{2}}
$$

cf. Eqs. (10). Varying $|C|^{2}$, one can tune the eigenfrequencies in the nonlinear case as per 
Models of spin-orbit coupled oligomers

Eq. (12), cf. Eq. (10). The norm of the nonlinear modes depends on $\lambda$, and can be found as

$$
P=-2 \mu \pm \sqrt{1+4 \lambda^{2}}
$$

To analyze the stability of the stationary nonlinear solutions, we apply the LSA, adding small arbitrary complex-valued perturbation $\delta \phi_{0,1}^{ \pm}$to the stationary modes. The perturbed solution is substituted into Eqs. (5)-(6), which are then linearized with respect to the small perturbations. The resulting linear system is then reduced to the EV problem with matrix

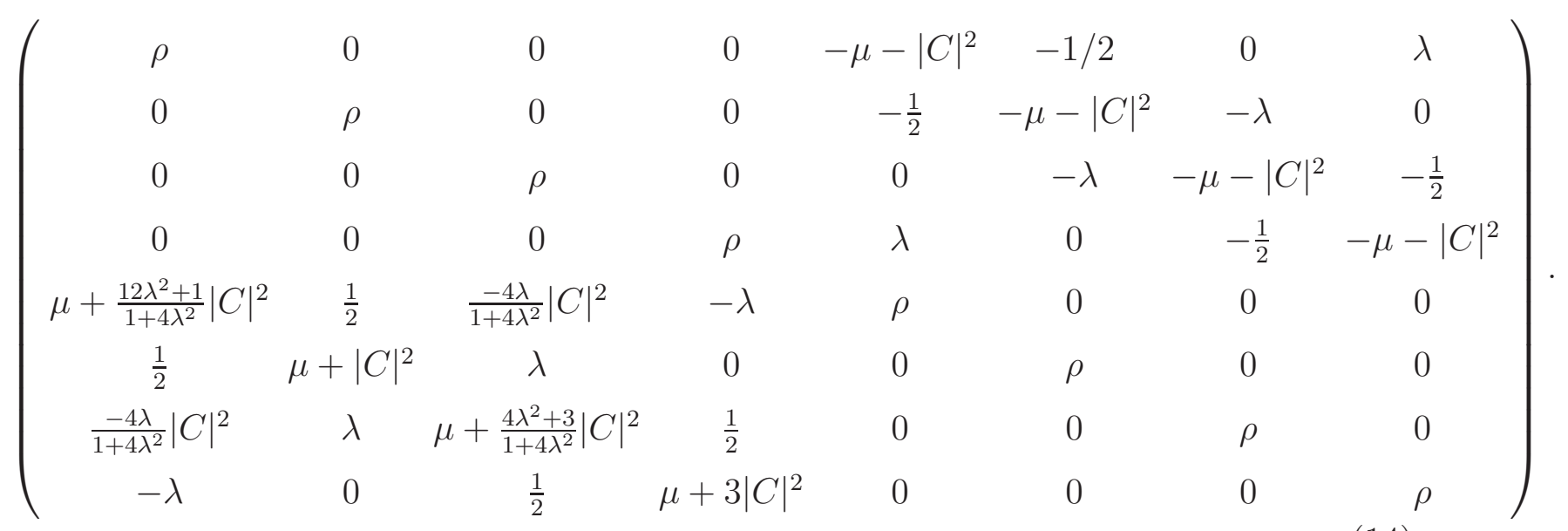

Eigenvalues of this matrix can be found in an exact form:

$$
\begin{gathered}
\rho_{1,2,3,4}=0 \\
\rho_{5,6}= \pm i \sqrt{1+4 \lambda^{2}} \\
\rho_{7,8}= \pm\left(1+4 \lambda^{2}\right)^{1 / 4} \sqrt{2\left(\mu-\sqrt{1+4 \lambda^{2}}\right)}
\end{gathered}
$$

where $|C|^{2}$ is substituted by $-\mu \pm \frac{1}{2} \sqrt{1+4 \lambda^{2}}$, as per Eq. (12).

As it follows from Eq. (12), the present stationary dimer solutions exist in the region of $-\infty<\mu \leq \frac{1}{2} \sqrt{1+4 \lambda^{2}}$, hence all EVs (15) have zero real parts, i.e., all the stationary solutions are stable. Direct simulations corroborate this conclusion, by displaying stable evolution of perturbed stationary solutions (not shown here in detail).

While each eigenvector from set (11) does not feature any intrinsic symmetry, linear combinations of the eigenvectors, taken as

$$
\left(\mathbf{v}_{12}\right)_{ \pm}=\mathbf{u}_{\mathbf{2}} \pm i \mathbf{u}_{1}, \quad\left(\mathbf{v}_{34}\right)_{ \pm}=\mathbf{u}_{\mathbf{4}} \pm i \mathbf{u}_{\mathbf{3}}
$$


produce solutions which obey the symmetry restriction defined by Eq. (2). As said above, it corresponds to the special case when the system is reduced to the single-component DNLSE (3), with two sites. Unlike the eigenvectors presented by the individual eigenmodes, these combinations, shown in Fig. 2, extend as nonlinear states even in the case of the nonManakov's nonlinearity, $\kappa \neq 1$, with the nonlinearly shifted eigenfrequencies $\mu=-(1+$ $\kappa)|C|^{2} \pm \frac{1}{2} \sqrt{1+4 \lambda^{2}}$ [note that sign \pm here has the same meaning as in Eq. (12), but not as in Eq. (16)]. The norm of the mode can be found as $P=-2 /(1+\kappa)\left(-2 \mu \pm \sqrt{1+4 \lambda^{2}}\right)$. The respective LSA and direct simulations confirm the stability of combined modes (16) in their existence region.

It is relevant to mention that the availability of the exact solutions for all the linear eigenmodes, as well as for some of the nonlinear ones, makes the use of the well-known method of the continuation from the anticontinuum limit ${ }^{30}$ (which corresponds to uncoupled lattices) unnecessary in the present case, both for the dimers and higher-order oligomers considered below.

\section{THE SPIN-ORBIT-COUPLED TRIMER}

The SO-coupled trimer string can be realized by adding an extra site $n=-1$ to the dimer. In this case, the dynamics is described by the following system of equations:

$$
\begin{gathered}
i \frac{d \phi_{-1}^{+}}{d t}=-\frac{1}{2} \phi_{0}^{+}-\left(\left|\phi_{-1}^{+}\right|^{2}+\kappa\left|\phi_{-1}^{-}\right|^{2}\right) \phi_{-1}^{+}+\lambda \phi_{0}^{-}, \\
i \frac{d \phi_{-1}^{-}}{d t}=-\frac{1}{2} \phi_{0}^{-}-\left(\left|\phi_{-1}^{-}\right|^{2}+\kappa\left|\phi_{-1}^{+}\right|^{2}\right) \phi_{-1}^{-}-\lambda \phi_{0}^{+} \\
i \frac{d \phi_{0}^{+}}{d t}=-\left(\frac{1}{2} \phi_{-1}^{+}+\frac{1}{2} \phi_{+1}^{+}\right)-\left(\left|\phi_{0}^{+}\right|^{2}+\kappa\left|\phi_{0}^{-}\right|^{2}\right) \phi_{0}^{+}+\lambda\left(-\phi_{-1}^{-}+\phi_{+1}^{-}\right), \\
i \frac{d \phi_{0}^{-}}{d t}=-\left(\frac{1}{2} \phi_{-1}^{-}+\frac{1}{2} \phi_{+1}^{-}\right)-\left(\left|\phi_{0}^{-}\right|^{2}+\kappa\left|\phi_{0}^{+}\right|^{2}\right) \phi_{0}^{-}+\lambda\left(\phi_{-1}^{+}-\phi_{+1}^{+}\right) \\
i \frac{d \phi_{+1}^{+}}{d t}=-\frac{1}{2} \phi_{0}^{+}-\left(\left|\phi_{+1}^{+}\right|^{2}+\kappa\left|\phi_{+1}^{-}\right|^{2}\right) \phi_{+1}^{+}-\lambda \phi_{0}^{-} \\
i \frac{d \phi_{+1}^{-}}{d t}=-\frac{1}{2} \phi_{0}^{-}-\left(\left|\phi_{+1}^{-}\right|^{2}+\kappa\left|\phi_{+1}^{+}\right|^{2}\right) \phi_{+1}^{-}+\lambda \phi_{0}^{+} .
\end{gathered}
$$


Models of spin-orbit coupled oligomers

Equations (17)-(19) conserve the corresponding energy,

$$
\begin{gathered}
E_{-1,0 .+1}=-\operatorname{Re}\left[\left(\phi_{-1}^{+}\right)^{*} \phi_{0}^{+}+\left(\phi_{0}^{+}\right)^{*} \phi_{+1}^{+}+\left(\phi_{-1}^{-}\right)^{*} \phi_{0}^{-}+\left(\phi_{0}^{-}\right)^{*} \phi_{+1}^{-}\right] \\
-\frac{1}{2}\left(\left|\phi_{-1}^{+}\right|^{4}+\left|\phi_{0}^{+}\right|^{4}+\left|\phi_{+1}^{+}\right|^{4}+\left|\phi_{-1}^{-}\right|^{4}+\left|\phi_{0}^{-}\right|^{4}+\left|\phi_{+1}^{-}\right|^{4}\right)-\kappa\left(\left|\phi_{-1}^{+}\right|^{2}\left|\phi_{-1}^{-}\right|^{2}+\left|\phi_{0}^{+}\right|^{2}\left|\phi_{0}^{-}\right|^{2}+\left|\phi_{+1}^{+}\right|^{2}\left|\phi_{+1}^{-}\right|^{2}\right) \\
+\lambda\left[\left(\phi_{-1}^{+}\right)^{*} \phi_{0}^{-}-\left(\phi_{0}^{+}\right)^{*}\left(\phi_{-1}^{-}-\phi_{+1}^{-}\right)-\left(\phi_{+1}^{+}\right)^{*} \phi_{0}^{-}-\left(\phi_{-1}^{-}\right)^{*} \phi_{0}^{+}+\left(\phi_{0}^{-}\right)^{*}\left(\phi_{-1}^{+}-\phi_{+1}^{+}\right)+\left(\phi_{+1}^{-}\right)^{*} \phi_{0}^{+}\right],
\end{gathered}
$$

and the total norm,

$$
P=\sum_{+,-}\left(\left|\phi_{-1}^{ \pm}\right|^{2}+\left|\phi_{0}^{ \pm}\right|^{2}+\left|\phi_{+1}^{ \pm}\right|^{2}\right)
$$

Assuming the usual form of stationary solutions,

$$
\phi_{-1,0,+1}^{ \pm}=\exp (-i \mu t) u_{-1,0,+1}^{ \pm}
$$

and substituting this in the linearized version of Eqs. (17)-(19), the following three doubledegenerate EVs are obtained:

$$
\mu_{1,2}^{(0)}=0, \mu_{3,4}^{(0)}=\sqrt{\frac{1}{2}+2 \lambda^{2}}, \mu_{5,6}^{(0)}=-\sqrt{\frac{1}{2}+2 \lambda^{2}} .
$$

The eigenvectors that correspond to $\mu_{1,2}^{(0)}=0$ are

$$
\begin{aligned}
& \mathbf{u}_{\mathbf{1}}=\left\{u_{-1}^{+}, u_{0}^{+}, u_{1}^{+}, u_{-1}^{-}, u_{0}^{-}, u_{1}^{-}\right\}=C\left\{1,0, \frac{4 \lambda^{2}-1}{1+4 \lambda^{2}}, 0,0, \frac{4 \lambda}{1+4 \lambda^{2}}\right\}, \\
& \mathbf{u}_{\mathbf{2}}=\left\{u_{-1}^{+}, u_{0}^{+}, u_{1}^{+}, u_{-1}^{-}, u_{0}^{-}, u_{1}^{-}\right\}=C\left\{\frac{4 \lambda^{2}-1}{1+4 \lambda^{2}}, 0,1,-\frac{4 \lambda}{1+4 \lambda^{2}}, 0,0\right\},
\end{aligned}
$$

see Fig. 3. Note that these eigenstates do not feature any symmetry. On the other hand, solutions of the linearized version system (17)-(19), obeying symmetry restrictions $u_{-1}^{+}=$ $u_{+1}^{+}, u_{-1}^{-}=-u_{+1}^{-}$and $u_{0}^{-}=0$, can be obtained as the linear combination of these two eigenvectors, $\mathbf{u}_{\mathbf{1}}+\mathbf{u}_{\mathbf{2}}$, see Fig. 3(c).

Eigenmodes (24), as well as their combination $\mathbf{u}_{\mathbf{1}}+\mathbf{u}_{\mathbf{2}}$, remain valid solutions of the nonlinear trimer system, but only in the above-mentioned Manakov's case, $\kappa=1$, cf. the similar result obtained above for the dimers. The respective nonlinear shift of the chemical potential, $\mu$, and the total norm,

$$
P=-2 \mu,
$$

do not depend on the SO-coupling parameter $\lambda$ (recall we consider the solution which has $\mu=0$ in the linear limit). The (dynamically stable) nonlinear extension of eigenvectors that 
Models of spin-orbit coupled oligomers

a)

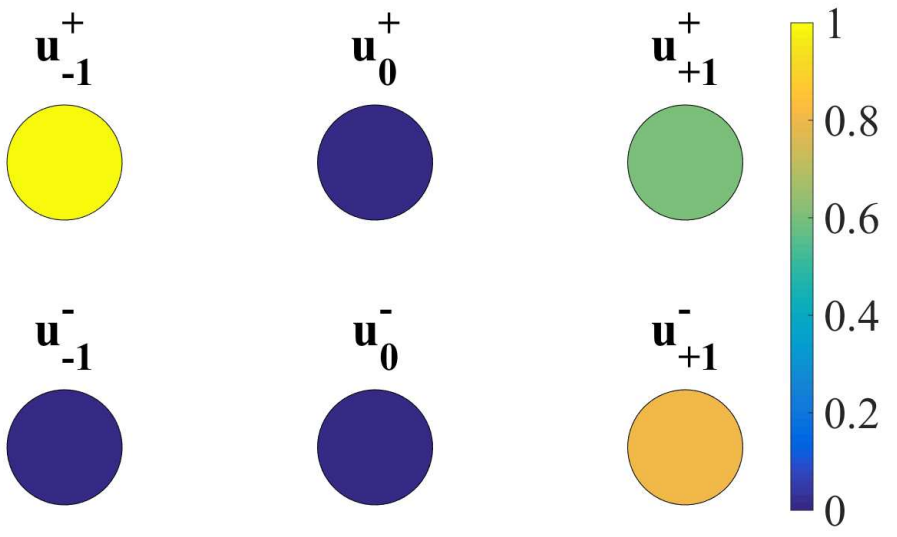

b)
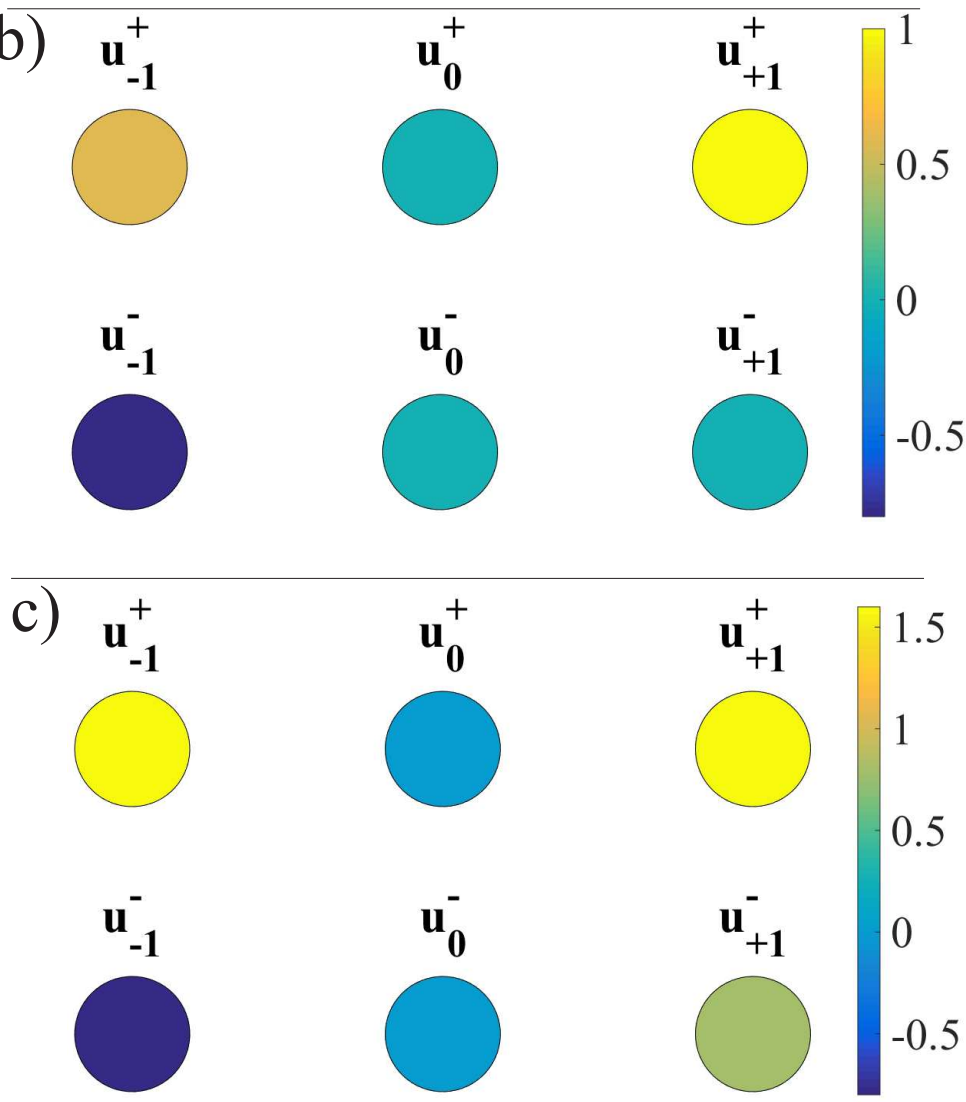

FIG. 3. (a,b) Eigenvectors of the linearized trimer system that correspond to $\mu_{1,2}^{(0)}=0$, and (c) their linear combination, $\mathbf{u}_{\mathbf{1}}+\mathbf{u}_{\mathbf{2}}$, at $\lambda=1$.

correspond to $\mu_{5,6}^{(0)}$, see Eq. (23) , is reported separately in Section VI, as it can be found solely in a numerical form.

The stability of these solutions of the nonlinear system with the Manakov's nonlinearity, $\kappa=1$, was analyzed by means of the LSA, following the same procedure as in the case of the dimer. An instability, i.e., positive real parts of the corresponding stability EVs, are 


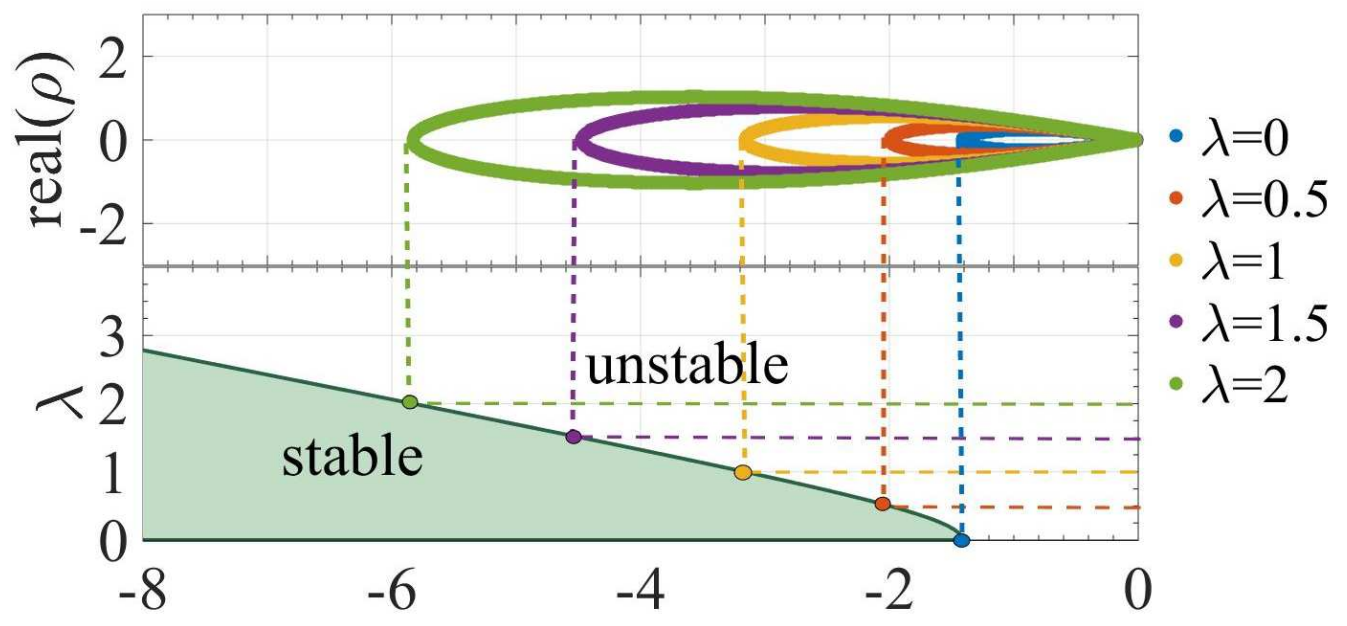

FIG. 4. (Top) Numerically obtained real parts of stability eigenvalues $\rho$ for the nonlinear trimer states given by Eq. (24), vs. $\mu$ for solutions $\mathbf{u}_{1}, \mathbf{u}_{2}$ from Eq. (24) at different values of $\lambda$. (Bottom) The corresponding stability diagram, in the $(\mu, \lambda)$ plane, with the stability boundary given by Eq. (26).

found, in an analytical form (confirmed by the numerical calculation), at

$$
\mu>\mu_{\mathrm{cr}}^{(\text {trimer })} \equiv-2 \sqrt{\frac{1}{2}+2 \lambda^{2}}
$$

see Fig. 4. Direct simulations corroborate the stability and instability of the trimer modes, as predicted by Eq. (26), see Fig. 5.

If we are looking for a solution subject to constraint (2), the SO-coupled trimer system can be reduced to the single-component DNLSE with three sites:

$$
\begin{aligned}
i \frac{d \phi_{-1}}{d t} & =-\left(\frac{1}{2}-i \lambda\right) \phi_{1}-(1+\kappa)\left|\phi_{-1}\right|^{2} \phi_{-1}, \\
i \frac{d \phi_{0}}{d t} & =-\left(\frac{1}{2}+i \lambda\right) \phi_{-1}-\left(\frac{1}{2}-i \lambda\right) \phi_{1}-(1+\kappa)\left|\phi_{0}\right|^{2} \phi_{0} \\
i \frac{d \phi_{1}}{d t} & =-\left(\frac{1}{2}+i \lambda\right) \phi_{0}-(1+\kappa)\left|\phi_{1}\right|^{2} \phi_{1} .
\end{aligned}
$$

In the linear limit, Eq. (27) yields the same EVs as given by Eq. (23). The eigenvector that corresponds to $\mathrm{EV} \mu^{(0)}=0$ is (see Fig. 6)

$$
\left\{u_{-1}^{+}, u_{0}^{+}, u_{1}^{+}, u_{-1}^{-}, u_{0}^{-}, u_{1}^{-}\right\}=C\left\{\frac{\left(4 \lambda^{2}-1\right)+4 i \lambda}{1+4 \lambda^{2}}, 0,1, \frac{-4 \lambda+i\left(4 \lambda^{2}-1\right)}{1+4 \lambda^{2}}, 0, i\right\} .
$$


Models of spin-orbit coupled oligomers
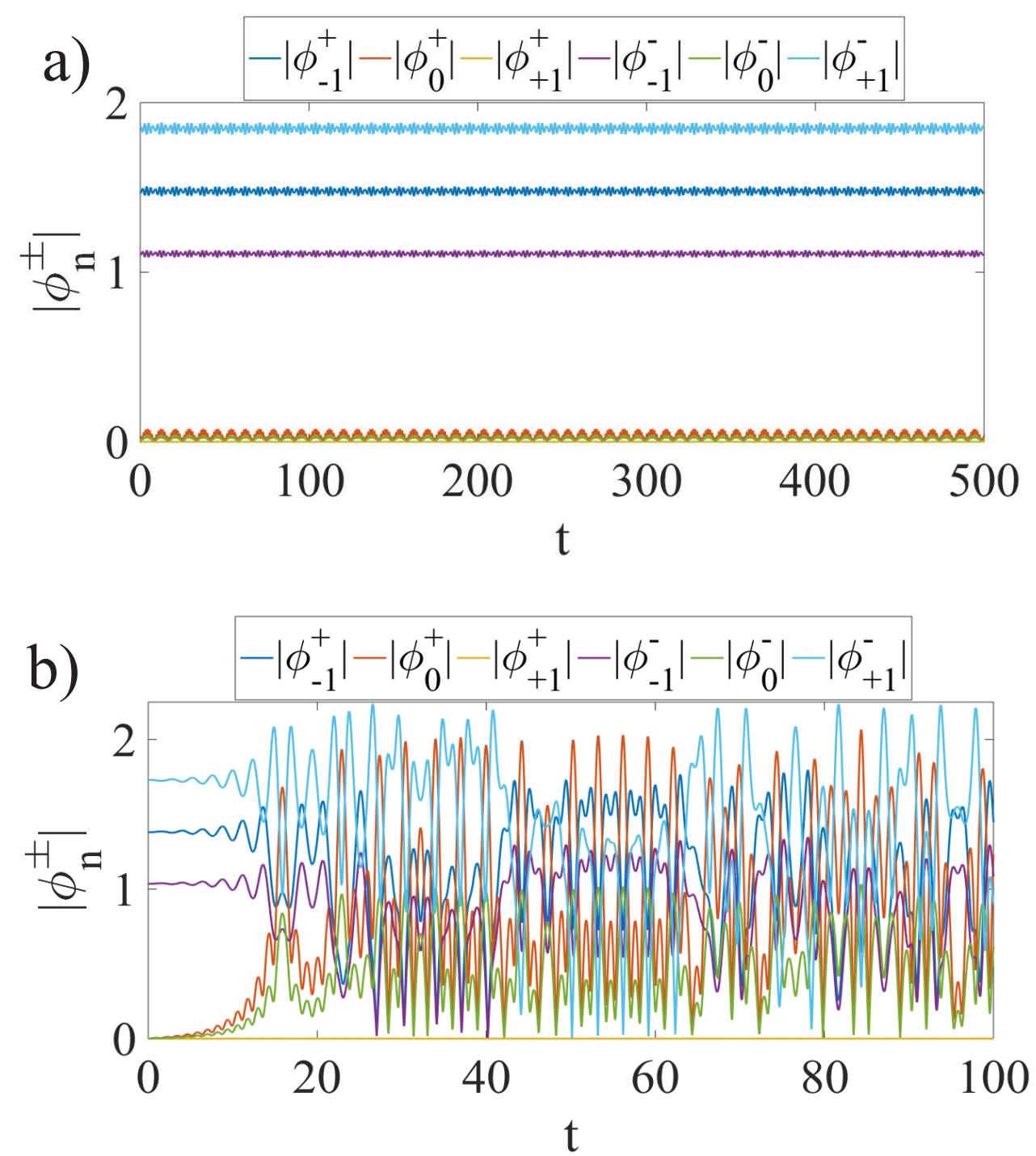

FIG. 5. (a) Amplitudes of a perturbed stable solution $\mathbf{u}_{\mathbf{1}}$ [see Eq. (24)], produced by simulations of the nonlinear trimer at $\mu=-3.5$ and $\lambda=1$. (b) The evolution of unstable trimer at $\mu=-3$ and $\lambda=1$.

It persists as an exact solution of the nonlinear system, even if $\kappa \neq 1$, with the respective nonlinear shift of the chemical potential, $\mu$, and the total norm $P=-4 \mu /(1+\kappa)$, which do not depend on the SO-coupling strength, $\lambda$.

The stability of the nonlinear solution (28) was analyzed by means of the LSA. The 
a)
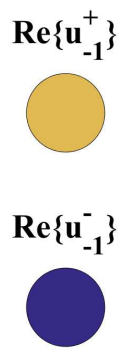

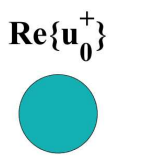

$\operatorname{Re}\left\{\mathbf{u}_{0}^{-}\right\}$

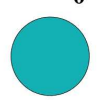

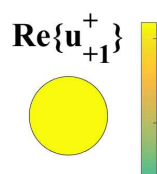

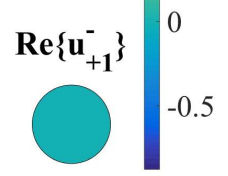

b)
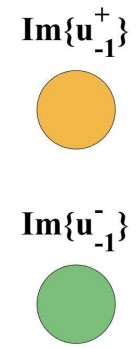
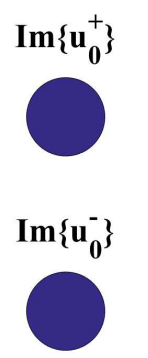

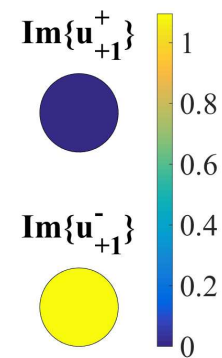

FIG. 6. The real and imaginary parts of the trimer eigenvector found as the solution of the nonlinear system at $\mu=-3, \kappa=1.5$, and $\lambda=1$.

following EVs have been thus obtained, in the analytical form:

$$
\begin{aligned}
& \rho_{1,2}=0, \\
& \rho_{3,4}= \pm i \frac{\sqrt{1+4 \lambda^{2}+\mu^{2}-\mu \sqrt{\mu^{2}-2\left(1+4 \lambda^{2}\right)}}}{\sqrt{2}}, \\
& \rho_{5,6}= \pm i \frac{\sqrt{1+4 \lambda^{2}+\mu^{2}+\mu \sqrt{\mu^{2}-2\left(1+4 \lambda^{2}\right)}}}{\sqrt{2}} .
\end{aligned}
$$

They feature an instability in the region identical to that defined by Eq. (26), i.e., the same as displayed for $\mathbf{u}_{\mathbf{1}}$ and $\mathbf{u}_{\mathbf{2}}$ in Fig. 4, with the difference that this solution exists at arbitrary $\kappa$. Direct simulations confirm this stability prediction.

\section{THE SPIN-ORBIT-COUPLED TETRAMER AND PENTAMER}

The tetramer string, with four sites (eight degrees of freedom), can be built by adding a new site to the trimer. Following the same procedure as in the cases of the dimer and trimer, we find four double-degenerate EVs from the respective linearized system:

$$
\begin{aligned}
& \mu_{1,2}^{(0)}=-\frac{1}{4}(\sqrt{5}-1) \sqrt{1+4 \lambda^{2}}, \mu_{3,4}^{(0)}=\frac{1}{4}(\sqrt{5}-1) \sqrt{1+4 \lambda^{2}}, \\
& \mu_{5,6}^{(0)}=-\frac{1}{4}(\sqrt{5}+1) \sqrt{1+4 \lambda^{2}}, \mu_{7,8}^{(0)}=\frac{1}{4}(\sqrt{5}+1) \sqrt{1+4 \lambda^{2}} .
\end{aligned}
$$

The corresponding eigenvectors have been found too. Because the set of EVs (30) does not include $\mu=0$, the tetramer system does not produce any analytically available nonlinear mode, unlike the trimer states given by Eqs. (24) and (28). A numerically found (chiefly, unstable) nonlinear mode, originating from $\mu_{5,6}^{(0)}$ in Eq. (30), is reported in Section VI. 
The pentamer string, with ten degrees of freedom, is built as a chain of five sites, at $n=-2,-1,0,1,2$. Following the same procedure as above, five double-degenerate EV branches are found for the linearized pentamer:

$$
\begin{aligned}
\mu_{1,2}^{(0)} & =0 \\
\mu_{3,4}^{(0)} & =\frac{1}{2} \sqrt{1+4 \lambda^{2}}, \\
\mu_{5,6}^{(0)} & =-\frac{1}{2} \sqrt{1+4 \lambda^{2}}, \\
\mu_{7,8}^{(0)} & =\frac{\sqrt{3}}{2} \sqrt{1+4 \lambda^{2}}, \\
\mu_{9,10}^{(0)} & =-\frac{\sqrt{3}}{2} \sqrt{1+4 \lambda^{2}} .
\end{aligned}
$$

Eigenvectors that correspond to $\mu_{1,2}^{(0)}=0$ are

$$
\begin{aligned}
\mathbf{u}_{\mathbf{1}} & =\left\{u_{-2}^{+}, u_{-1}^{+}, u_{0}^{+}, u_{1}^{+}, u_{2}^{+}, u_{-2}^{-}, u_{-1}^{-}, u_{0}^{-}, u_{1}^{-}, u_{2}^{-}\right\}= \\
& =C\left\{\frac{4 \lambda^{2}-1}{1+4 \lambda^{2}}, 0,1,0, \frac{4 \lambda^{2}-1}{1+4 \lambda^{2}},-\frac{4 \lambda}{1+4 \lambda^{2}}, 0,0,0, \frac{4 \lambda}{1+4 \lambda^{2}}\right\}, \\
\mathbf{u}_{2} & =\left\{u_{-2}^{+}, u_{-1}^{+}, u_{0}^{+}, u_{1}^{+}, u_{2}^{+}, u_{-2}^{-}, u_{-1}^{-}, u_{0}^{-}, u_{1}^{-}, u_{2}^{-}\right\}= \\
& =C\left\{-\frac{\left(4 \lambda^{2}-1\right)^{2}-16 \lambda^{2}}{\left(1+4 \lambda^{2}\right)^{2}}, 0,-\frac{4 \lambda^{2}-1}{1+4 \lambda^{2}}, 0,-1, \frac{8 \lambda\left(4 \lambda^{2}-1\right)}{\left(1+4 \lambda^{2}\right)^{2}}, 0, \frac{4 \lambda}{1+4 \lambda^{2}}, 0,0\right\},
\end{aligned}
$$

see Fig. 7. Note that eigenvector $\mathbf{u}_{1}$ obeys constraints $u_{-n}^{+}=u_{n}^{+}, u_{-n}^{-}=-u_{n}^{-}$, and $u_{0}^{-}=0$.

Like in the trimer case, the eigenvectors that correspond to $\mu_{1,2}^{(0)}=0$ extend as solutions of the full nonlinear system, but solely under the Manakov's condition, $\kappa=1$. Once again, their nonlinear frequency shift $\mu$ and total norm,

$$
P=-3 \mu
$$

do not depend on $\lambda$, cf. a similar relation (25) for the trimers. The LSA, implemented for these nonlinear states, predicts instability at

$$
\mu>\mu_{\mathrm{cr}}^{(\text {pentamer })} \equiv-\sqrt{3+12 \lambda^{2}}
$$

and stability at $\mu<\mu_{\mathrm{cr}}^{\text {(pentamer) }}[$ cf. Eq. (26) ], see Fig. 8. This prediction has been confirmed by direct simulations (not shown here in detail, as the results are quite similar to those displayed above for stable and unstable trimers in Fig. 51).

Looking for solutions that satisfy constraint (2), the SO-coupled pentamer can be reduced to the single-component chain with five sites. The respective linearized system yields the 
Models of spin-orbit coupled oligomers

a)
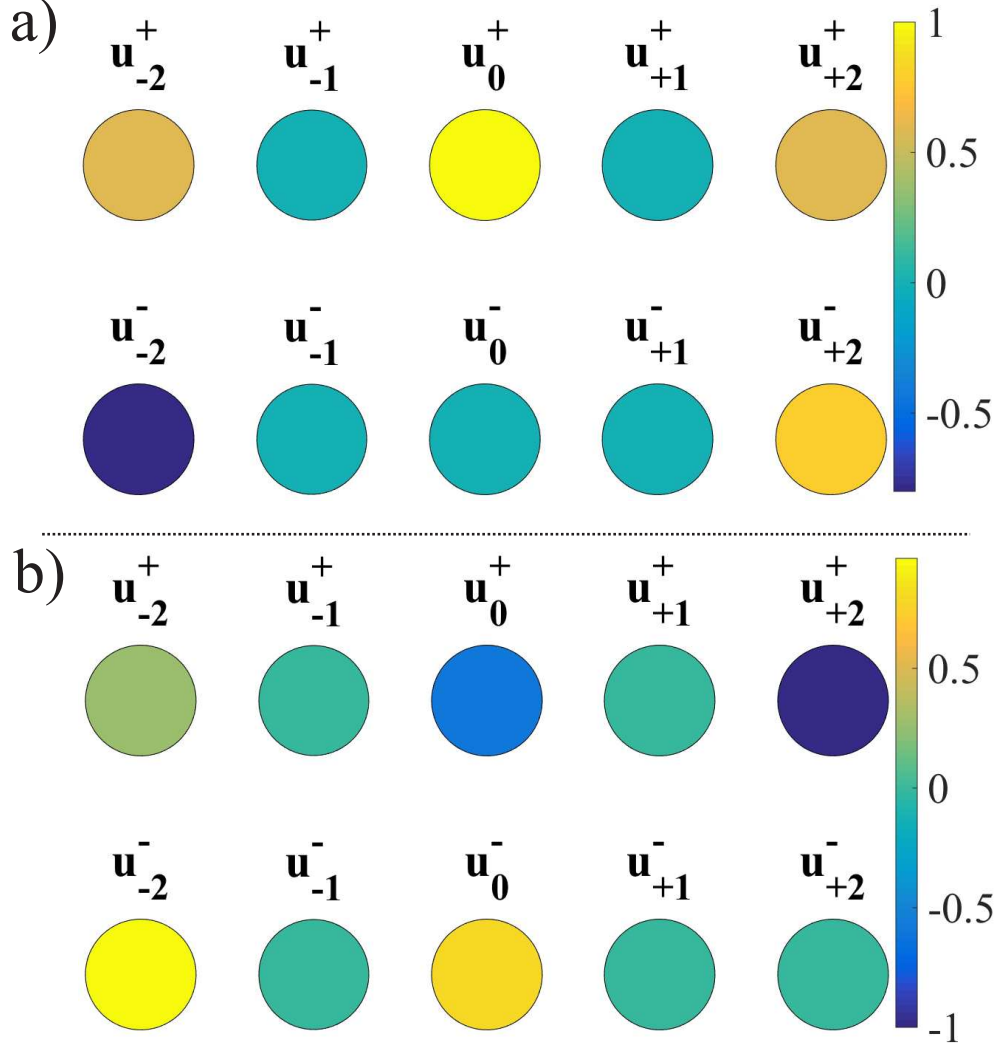

FIG. 7. Pentamer's eigenvectors (32) that correspond to $\mu_{1,2}^{(0)}=0$, at $\lambda=1$.

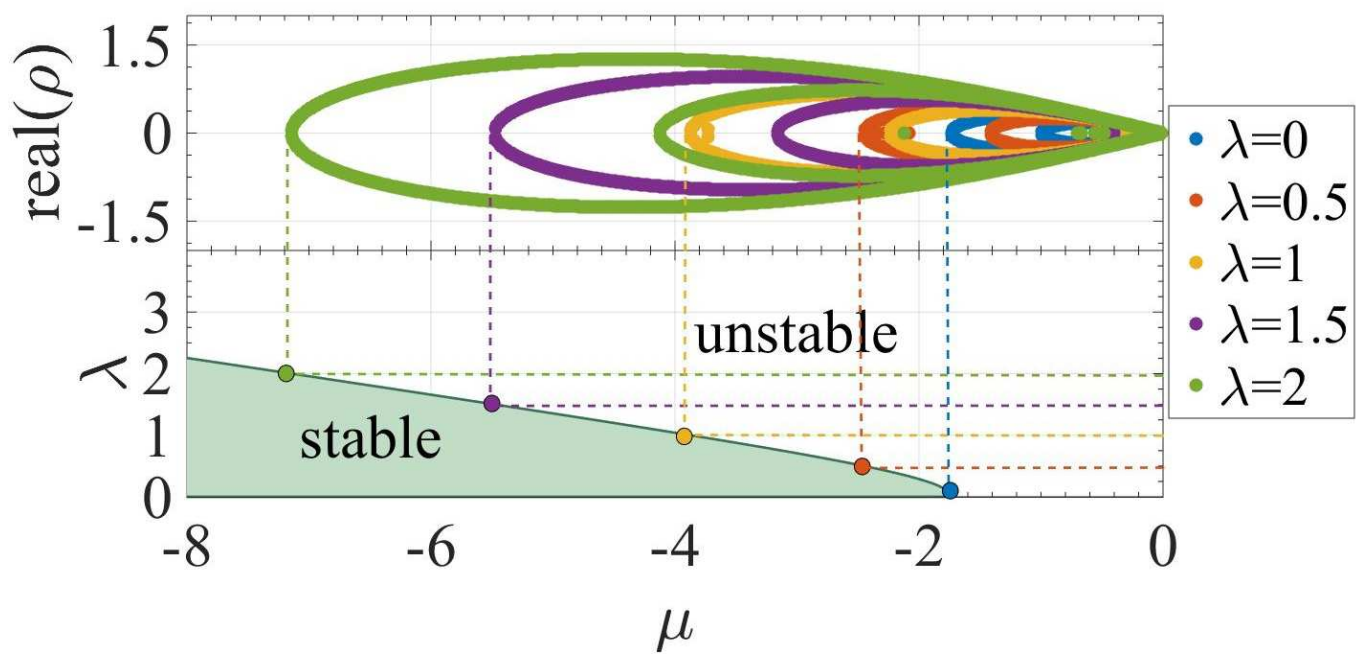

FIG. 8. (Top) Numerically obtained real parts of stability eigenvalues $\rho$ vs. $\mu$ for nonlinear pentamer solutions $\mathbf{u}_{\mathbf{1}}$ and $\mathbf{u}_{\mathbf{2}}$, given by Eq. (32). (Bottom) The corresponding stability diagram, with the stability boundary given by Eq. (34). 
a)
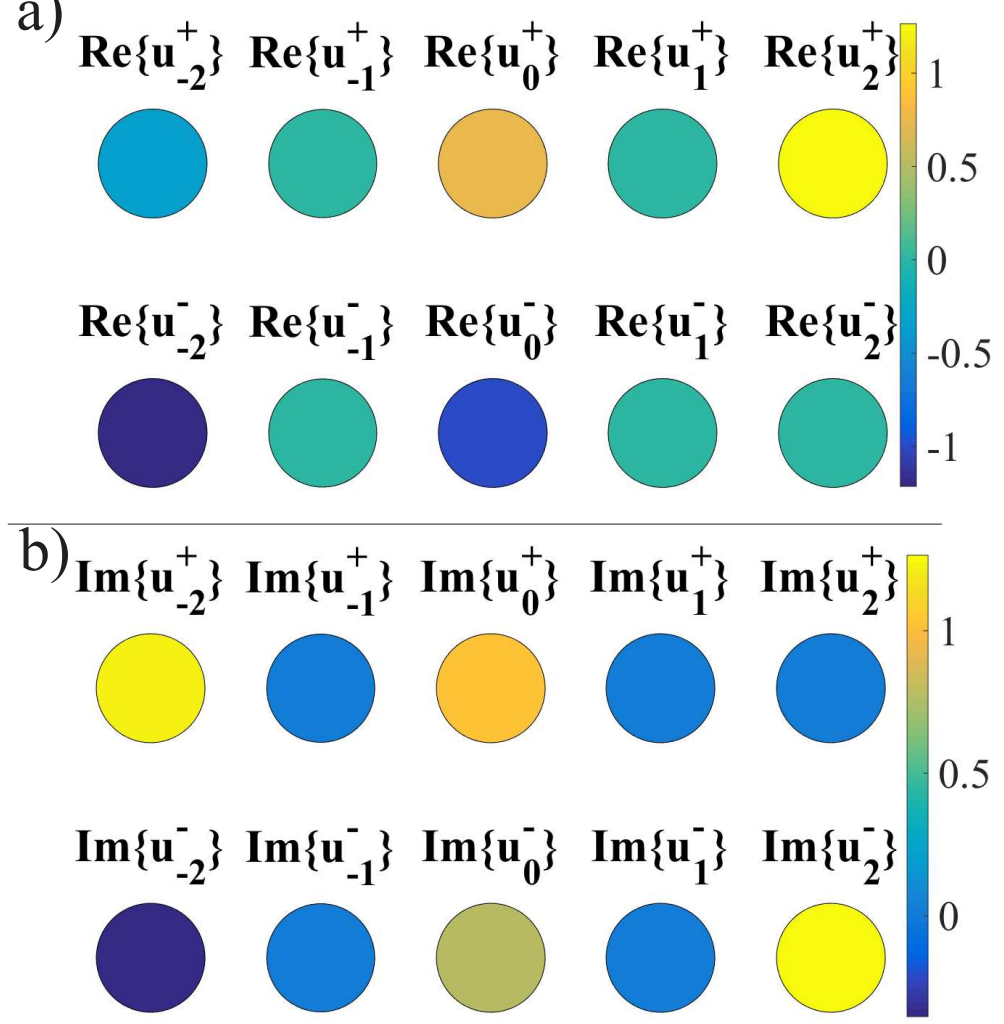

FIG. 9. The real and imaginary parts of eigenvector (32) of the nonlinear pentamer system, that satisfies constraint (2) for $\mu=-4, \kappa=1.5$, and $\lambda=1$.

same eigenvalues as produced above in Eq. (31). The eigenvector that corresponds to $\mu^{(0)}=0$ is

$$
\begin{gathered}
\left\{u_{-2}^{+}, u_{-1}^{+}, u_{0}^{+}, u_{1}^{+}, u_{2}^{+}, u_{-2}^{-}, u_{-1}^{-}, u_{0}^{-}, u_{1}^{-}, u_{2}^{-}\right\}= \\
=C\left\{\frac{(2 \lambda+i)^{4}}{\left(1+4 \lambda^{2}\right)^{2}}, 0, \frac{(2 \lambda+i)^{2}}{1+4 \lambda^{2}}, 0,1, \pm i \frac{(2 \lambda+i)^{4}}{\left(1+4 \lambda^{2}\right)^{2}}, 0, \pm i \frac{(2 \lambda+i)^{2}}{1+4 \lambda^{2}}, 0, \pm i\right\},
\end{gathered}
$$

see Fig. 9. This eigenvector keeps its form in the presence of the nonlinearity, irrespective of the value of the nonlinear parameter $\kappa$ (i.e., in the general non-Manakov case). The corresponding nonlinear shift of the chemical potential, $\mu$, and the total norm,

$$
P=-6 \mu /(1+\kappa)
$$

do not depend on the SO-coupling strength, $\lambda$, similar to what was found above for other solutions subject to constraint (22), cf. Eqs. (25) and (33). The LSA demonstrates that the nonlinear solutions, which originate from the linear eigenvector (35), precisely share the 
Models of spin-orbit coupled oligomers

stability properties with their counterparts, $\mathbf{u}_{\mathbf{1}}$ and $\mathbf{u}_{\mathbf{2}}$, see Fig. 8. This prediction has been confirmed by direct simulations.

The linear mode corresponding to $\mu_{9,10}^{(0)}$ in Eq. (31) can numerically extended into a stable nonlinear state, as shown in the following section.

\section{NONLINEAR MODES ORIGINATING FROM NONZERO EIGENVALUES IN THE LINEAR LIMIT}

In the previous section we have shown that the linear eigenmodes associated with eigenvalue $\mu^{(0)}=0$ persist in nonlinear trimer and pentamer systems without any change of the shape. The increase of the nonlinearity strength only affects their stability, in the sense that, above a certain value of the strength, these modes become unstable. The other property of these modes is the independence of the norm on the SO-coupling parameter.

Using numerical methods, we have found other families of nonlinear states in all the considered $N$-mers, which originate, in the linear limit, from trimers, tetramers, and pentamer eigenmodes associated with $\mathrm{EVs} \mu_{5,6}^{(0)}, \mu_{5,6}^{(0)}$, and $\mu_{9,10}^{(0)}$, respectively [see Eqs. (23), (30), and (31)]. As an example of these modes, we first present, in Fig. 10, numerically generated nonlinear solutions in the trimer system, which feature even and odd $(+$ and -$)$ spinor components, respectively:

$$
u_{-1}^{(+)}=u_{+1}^{(+)}, u_{-1}^{(-)}=-u_{+1}^{(-)}, u_{0}^{(-)}=0
$$

In general, the families of the nonlinear trimer solutions, which exist for different values of $\lambda$ and arbitrary values of $\kappa$, are characterized by dependencies of the total norm on the chemical potential, $P(\mu)$, as shown in Fig. 11. These nonlinear trimers are stable in their entire existence region, according to the LSA, which is confirmed by direct simulations. In this connection, it is relevant to mention that all $P(\mu)$ curves in Fig. 11, as well as in Figs. 13 and 16 displayed below for nonlinear tetramer and pentamer modes, satisfy the well-known Vakhitov-Kolokolov (VK) stability criterion, $d P / d \mu<0$, which is a necessary condition (but, generally speaking, is not a sufficient one - see the situation for the tetramers presented below) for the stability of solitons supported by attractive nonlinearities 31 .

In the tetramer system, we have numerically found a family of nonlinear solutions, which 
Models of spin-orbit coupled oligomers

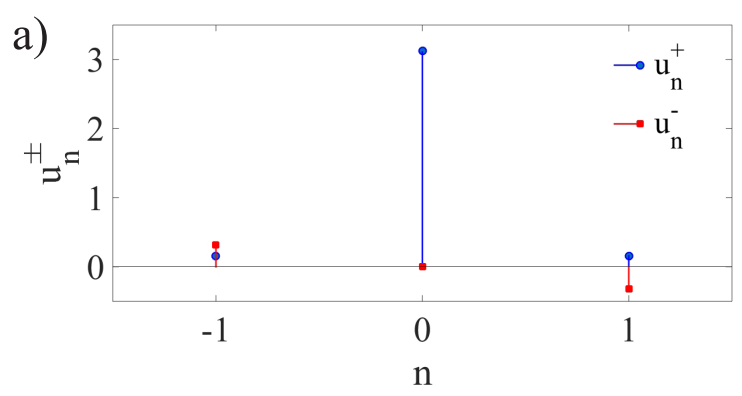

b)

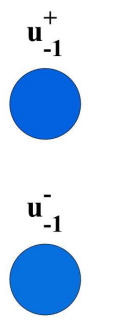

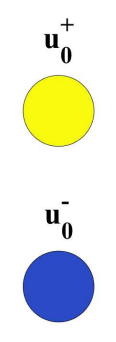

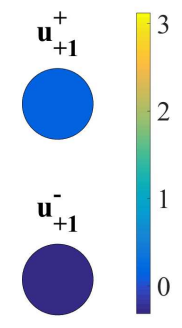

FIG. 10. The numerically found stable solution for the nonlinear trimer mode, which, in the linear limit, originates from the linear eigenmode corresponding to $\mu_{5,6}^{(0)}$ in Eq. (23). It is obtained for $\mu=-10, \kappa=0.5$, and $\lambda=1$. (a) The amplitude profile of the solution. (b) Its schematic structure.

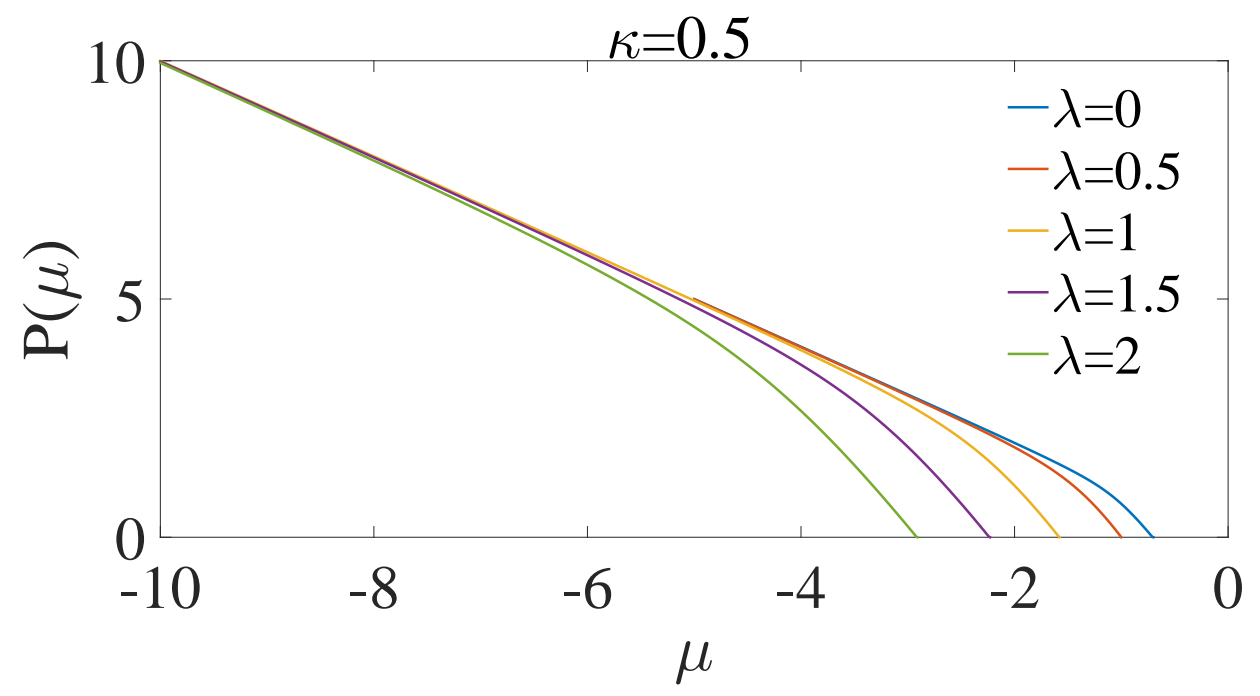

FIG. 11. $P(\mu)$ curves for the families of nonlinear trimer modes, originating from $\mu_{5,6}^{(0)}$ in Eq. (23) , at different values of $\lambda$, and $\kappa=0.5$.

obey the symmetry relations in one spinor component,

$$
u_{0}^{(+)}=u_{1}^{(+)}, u_{-1}^{(+)}=u_{2}^{(+)}
$$

while the other component is asymmetric, see Fig. 12, $P(\mu)$ dependencies for the tetramer nonlinear solutions are shown in Fig. 13 for different values of the SO-coupling strength, $\lambda$.

The LSA analysis indicates instability of the nonlinear tetramer modes in their nearly whole existence region, in spite of the fact that the $P(\mu)$ curves satisfy the VakhitovKolokolov criterion in Fig. 13. Narrow stability windows open only for the Manakov's 

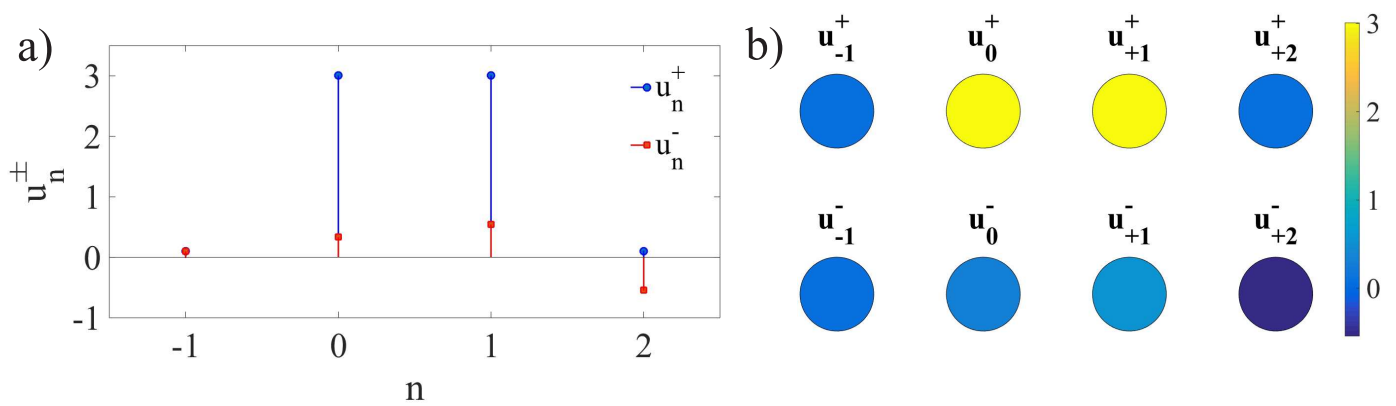

FIG. 12. An unstable nonlinear tetramer solution obtained at $\mu=-10, \kappa=0.5$, and $\lambda=1$.

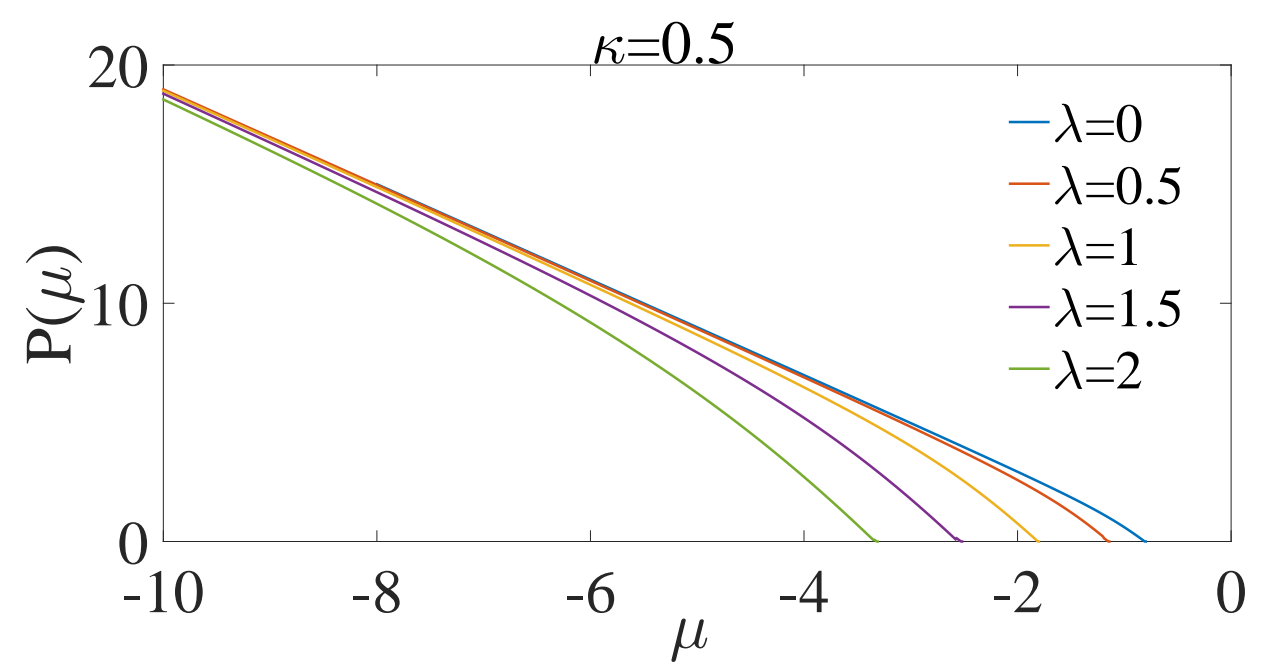

FIG. 13. $P(\mu)$ curves for the nonlinear tetramer modes, originating from $\mu_{5,6}^{(0)}$ in Eq. (30), for different values of $\lambda$, and $\kappa=0.5$.

type of the nonlinearity $(\kappa=1)$. As an example, the EV spectrum of small perturbations for the tetramer with $\lambda=1$ is displayed in Fig. 14 for $\kappa=0,0.5$, and 1. A stability interval, $-2.7<\mu<0$, is found at $\kappa=1$.

Finally, Fig. 15 demonstrates that nonlinear pentamer modes, originating, in the linear limit, from EV $\mu_{9,10}^{(0)}$ in Eq. (31), obey the same symmetry as their trimer counterparts (cf. Eq. (37)),

$$
u_{-1,-2}^{(+)}=u_{+1,+2}^{(+)}, u_{-1,-2}^{(-)}=-u_{+1,+2}^{(-)}, u_{0}^{(-)}=0 .
$$

The corresponding $P(\mu)$ dependencies for certain values of $\lambda$ are displayed in Fig. 16. According to the LSA and direct simulations, the entire family of these nonlinear pentamer solutions is stable.

An essential difference of the nonlinear trimer and pentamer modes considered here from 


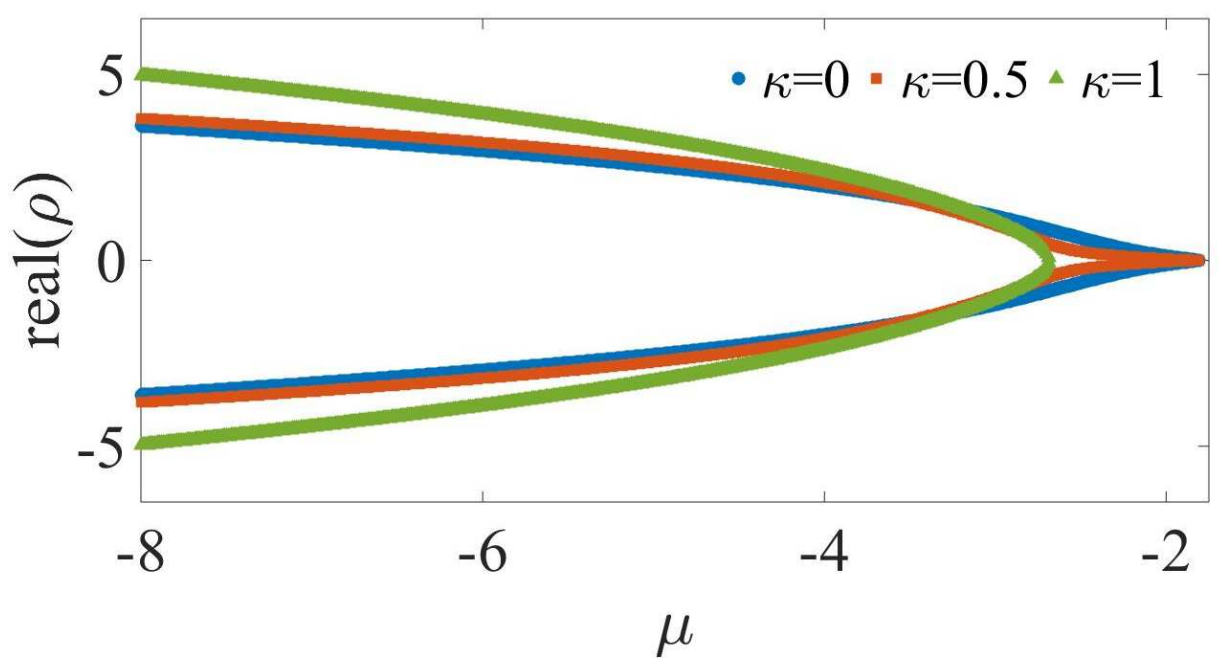

FIG. 14. Real parts of stability eigenvalues $\rho$ for the nonlinear tetramer modes vs. $\mu$ at $\kappa=0$ and 0.5 (blue and red lines, respectively), and $\kappa=1$ (the green line), while $\lambda=1$ is fixed. To avoid making the plot messy, the zero eigenvalue for $\kappa=1$ is not shown in the corresponding stability interval, $-2.7<\mu<0$.

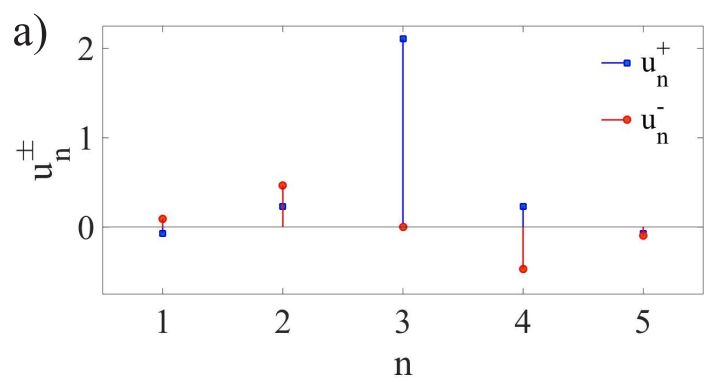

b)

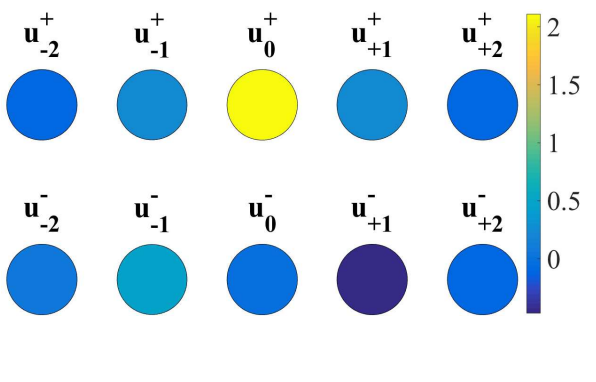

FIG. 15. A stable nonlinear pentamer solution numerically found for $\mu=-5, \kappa=0.5$, and $\lambda=1$. It obeys symmetry constraint (39).

those presented in the previous sections [see Eqs. (25), (33) and (36)], is that their total norm depends on the SO-coupling constant, $\lambda$. As a result, both types of the nonlinear trimer and pentamer modes may coexist at the same values of the chemical potential, $\mu$, but having different norms.

Finally, we have constructed families of linear and nonlinear 6- and 7- (hexa- and septa)mer modes. In the former case, they feature properties similar to those of the tetramers, while in the latter case the properties are similar to those of trimers and pentamers. Thus, the properties of oligomers are chiefly determined by the parity of the number of their sites. 
Models of spin-orbit coupled oligomers

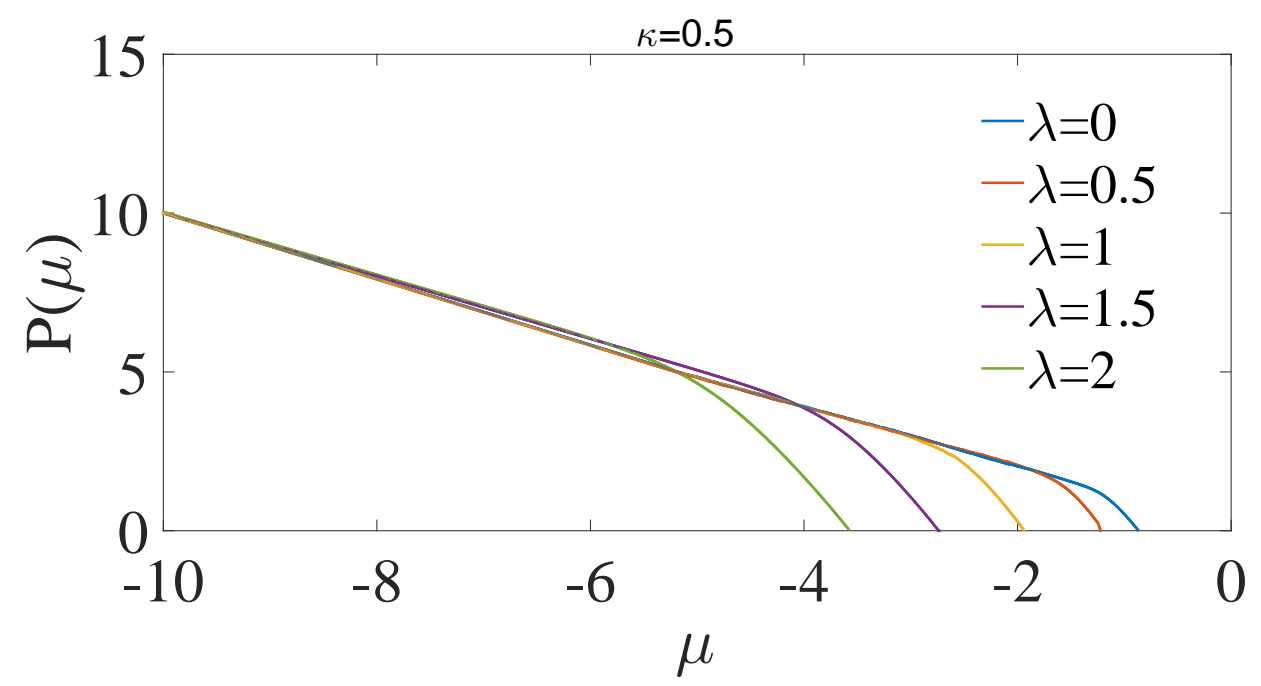

FIG. 16. $P(\mu)$ curves for families of the nonlinear pentamer states, originating, in the linear limit, from egenmodes corresponding to $\mu_{9,10}^{(0)}$ in Eq. (31), for indicated values of $\lambda$ and $\kappa=0.5$.

\section{CONCLUSION}

We have studied small linearly-shaped strings composed of two-component BEC droplets with the intrinsic pseudo-SO (spin-orbit) coupling and attractive nonlinearity, which can be implemented in current experiments with matter waves trapped in optical lattices. We have found eigenmodes of these systems, in analytical and numerical forms, and explored their stability by means of numerical methods, through the calculation of eigenfrequencies for small perturbations and direct simulations. The analysis has been performed for the dimers, trimers, tetramers, and pentamers.

In the dimer system, linear-eigenmode families can be generically extended as nonlinear modes only in the case of equal strengths of the intra- and inter-species attractive interactions (the Manakov's nonlinearity). The exception is the branch of the eigenmodes subject to constraint (2), which exists for arbitrary values of the nonlinearity parameters. The analysis has demonstrated that the families of nonlinear dimer modes are stable in their whole existence regions.

In the trimer and pentamer systems, only those eigenmodes whose eigenvalues are zero in the linear limit, $\mu=0$, can be extended, keeping their shape unaltered, to the nonlinear system. The eigenmodes which satisfy constraint (2) show properties similar to those of their dimer counterparts. Their existence region, in the presence of the nonlinearity, is bounded 
by $\mu<0$ (negative chemical potential) and does not depend on the SO strength, $\lambda$, while the stability boundary depends on $\lambda$. In all cases, the stability area of the nonlinearly continued eigenmodes shrinks with the increase of $\lambda$. On the contrary, in the tetramer system the nonlinear modes cannot be found in the exact form, because the respective linear spectrum does not include $\mu=0$.

In addition, in the trimer and pentamer systems the families of completely stable nonlinear modes are found (in the numerical form), which in the linear limit, originate from eigenmodes corresponding to $\mu \neq 0$. In the tetramer system, such nonlinear modes are found too. However, in the latter case those modes can be stable, in a narrow range of the system's parameters, solely with the Manakov's type of the nonlinearity.

As concerns possibilities for extension of the analysis, one of them is to analyze a possible supersolid phase in the truncated lattice, as suggested by the recent experiment performed in the SO-coupled BEC21.

\section{ACKNOWLEDGMENTS}

The authors acknowledge support from the Ministry of Education, Science and Technological Development of Republic of Serbia (project III45010). The work of B.A.M. was supported, in part, by grant No. 2015616 from the joint program in physics between the NSF and Binational (US-Israel) Science Foundation, and by grant No. 1286/17 from the Israel Science Foundation.

\section{REFERENCES}

${ }^{1}$ H. Sakaguchi, S. Shinnomoto, and Y. Kuramoto, "Local and global self-entrainment in oscillator lattices," Prog. Theor. Phys. 77, 1005-1010 (1987); H. Sakaguchi, "Cooperative phenomena in coupled oscillator systems under external fields," ibid. 79, 39-46 (1988).

${ }^{2}$ S. H. Strogatz, "Exploring complex networks," Nature 410, 268-276 (2001); R. Albert and A. L. Barabasi, "Statistical mechanics of complex networks," Rev. Mod. Phys. 74, 47-97 (2002); A. Arenas, A. Diaz-Guilera, J. Kurths, Y. Moreno, and C. S. Zhou, "Synchronization in complex networks," Phys. Rep. 469, 93-153 (2008); M. Barthelemy, Spatial networks, ibid. 499, 1-101 (2011). 
${ }^{3}$ M. L. Chiofalo, M. Polini, and M. P. Tosi, "Collective excitations of a periodic Bose condensate in the Wannier representation," Eur. Phys. J. D 11, 371-378 (2000); A. Smerzi, A. Trombettoni, P. G. Kevrekidis, and A. R. Bishop, "Dynamical superfluid-insulator transition in a chain of weakly coupled Bose-Einstein condensates," Phys. Rev. Lett. 89, 170402 (2002); G. L. Alfimov, P. G. Kevrekidis, V. V. Konotop, and M. Salerno, "Wannier functions analysis of the nonlinear Schrödinger equation with a periodic potential," Phys. Rev. E 66, 046608 (2002); A. Smerzi and A. Trombettoni, "Nonlinear tight-binding approximation for Bose-Einstein condensates in a lattice," Phys. Rev. A 68, 023613 (2003); G. H. Chong, W. H. Hai, and Q. T. Xie, "Controlling chaos in a weakly coupled array of BoseEinstein condensates," Phys. Rev. E 71, 016202 (2005); G. P. Zheng, J. Q. Liang, and W. M. Liu, "Phase diagram of two-species Bose-Einstein condensates in an optical lattice," Phys. Rev. A 71, 053608 (2005); S. Gopalakrishnan,B. L. Lev, and P. M. Goldbart, "Atomlight crystallization of Bose-Einstein condensates in multimode cavities: Nonequilibrium classical and quantum phase transitions, emergent lattices, supersolidity, and frustration," Phys. Rev. A 82, 043612 (2010).

${ }^{4}$ D. Jaksch, C. Bruder, J. I. Cirac, C. W. Gardiner, and P. Zoller, "Cold bosonic atoms in optical lattices," Phys. Rev. Lett. 81, 3108-3111 (1998); M. Greiner, O. Mandel, T. Esslinger, T. W. Hansch, and I. Bloch, "Quantum phase transition from a superfluid to a Mott insulator in a gas of ultracold atoms," Nature 415, 39-44 (2002); T. Stoferle, H. Moritz, C. Schori, M. Kohl, and T. Esslinger, "Transition from a strongly interacting 1D superfluid to a Mott insulator," Phys. Rev. Lett. 92, 130403 (2004); F. Gerbier, A. Widera, S. Folling, O. Mandel, T. Gericke, and I. Bloch, "Phase coherence of an atomic Mott insulator," Phys. Rev. Lett. 95, 050404 (2005); R. Fulton, A. I. Bishop, M. N. Shneider, and P. F. Barker, "Controlling the motion of cold molecules with deep periodic optical potentials," Nature Phys. 2, 465-468 (2006).

${ }^{5}$ T. Schumm, S. Hofferberth, L. M. Andersson, S. Wildermuth, S. Groth, I. Bar-Joseph, J. Schmiedmayer and P. Krüger, "Matter-wave interferometry in a double well on an atom chip", Nature Physics 1, 57 - 62 (2005).

${ }^{6}$ D. L. Campbell, G. Juzeliunas, and I. B. Spielman, "Realistic Rashba and Dresselhaus spin-orbit coupling for neutral atoms," Phys. Rev. A 84, 025602 (2001).

${ }^{7}$ Y. J. Lin, K. Jimenez-Garcia, and I. B. Spielman,"Spin-orbit-coupled Bose-Einstein condensates," Nature 471, 83(2011). 
Models of spin-orbit coupled oligomers

${ }^{8}$ V. Galitski, and I. B. Spielman," Spin-orbit coupling in quantum gases," Nature 494, 49 (2013).

${ }^{9}$ J-Y. Zhang, J.-Y. Zhang, S.-C. Ji, Z. Chen, L. Zhang, Z.-D. Du, B. Yan, G.-S. Pan, B. Zhao, Y. Deng, H. Zhai, S. Chen, and J.-W. Pan, "Collective dipole oscillation of a spin-orbit coupled Bose-Einstein condensate," Phys. Rev. Lett. 109, 115301(2012).

${ }^{10}$ C. Schmidt-Hattenberger, R. Mushall, U. Trutschel, and F. Lederer,"Nonlinear eigenmodes of a three-core fibre coupler," Optical and Quantum Electronics 24, 691-701 (1992).

${ }^{11}$ M. I. Molina and G. P. Tsironis, "Dynamics of self-trapping in the discrete nonlinear Schrödinger equation," Physica D 65, 267 (1993).

${ }^{12}$ N. N. Akhmediev and A. V. Buryak, "Soliton states and bifurcation phenomena in threecore nonlinear fiber couplers," J. Opt. Soc. Am. B 11, 804-809 (1994)

${ }^{13}$ J. C. Eilbeck, G. P. Tsironis and S. K. Turitsyn, "Stationary states in a doubly nonlinear trimer model of optical couplers," Physica Scripta 52, 386-387 (1995).

${ }^{14}$ A. B. Aceves, M. Santagiustina and C. De Angelis, " Analytical study of nonlinear-optical pulse dynamics in arrays of linearly coupled waveguides," J. Opt. Soc. Am. B 14, 1807-1815 (1996).

${ }^{15}$ A. B. Aceves and M. Santagiustina, "Bistable and tristable soliton switching in collinear arrays of linearly coupled waveguides," Phys. Rev. E 56, 1113-1123 (1997).

${ }^{16}$ M. Liu and K. S. Chiang, "Nonlinear switching of ultrashort pulses in multicore fibers," IEEE J. Quant. Elect. 47, 1499-1505 (2011).

${ }^{17}$ J. Hua, H. Zhou, K. S. Chiang, and S. R. Xiao, "Modulation instabilities in equilateral three-core optical fibers," J. Opt. Soc. Am. B 33, 2357-2367 (2016).

${ }^{18}$ D. Hennig, "The discrete self-trapping equation and the Painleve property," J. Phys. A 25, 1247 (1992).

${ }^{19}$ J. C. Eilbeck, P. S. Lomdahl, and A. C. Scott," The discrete self-trapping equation," Physica D 16, 318 (1985).

${ }^{20}$ J. Larson, J-P. Martikainen, A. Collin, E. Sjöqvist," Spin-orbit-coupled Bose-Einstein condensate in a tilted optical lattice", Phys. Rev. A 82, 043620 (2010); Y. Zhang and Ch. Zhang, "Bose-Einstein condensates in spin-orbit-coupled optical lattices: Flat bands and superfluidity", Phys. Rev. A 87, 023611 (2013); Y. V. Kartashov, V. V. Konotop, D. A. Zezyulin, and L. Torner, "Bloch Oscillations in Optical and Zeeman Lattices in the Presence of Spin-Orbit Coupling", Phys. Rew. Lett. 117, 215301 (2016). 
Models of spin-orbit coupled oligomers

${ }^{21}$ J-R. Li, J. Lee, W. Huang, S. Burchesky, B. Shteynas, F. Ç. Top, A. O. Jamison1 and W. Ketterle, "A stripe phase with supersolid properties in spin-orbit-coupled Bose-Einstein condensates", Nature 543, 91 (2017).

${ }^{22}$ H. Sakaguchi and B. A. Malomed, "Discrete and continuum composite solitons in BoseEinstein condensates with the Rashba spin-orbit coupling in one and two dimensions," Phys. Rev. E 90, 062922 (2014).

${ }^{23}$ M. Salerno, F. Kh. Abdullaev, "Symmetry breaking of localized discrete matter waves induced by spin-orbit coupling", Phys. Lett. A 379, 2252 (2015).

${ }^{24}$ R. Citro and A. Naddeo, "Spin-orbit coupled Bose-Einstein condensates in a double well", Eur. Phys. J. Special Topics 224, 503-518 (2015).

${ }^{25}$ P. P. Beličev, G. Gligorić, J. Petrović, A. Maluckov, Lj. Hadžievski, and B. A. Malomed, "Composite localized modes in discretized spin-orbit-coupled Bose-Einstein condensates," J. Phys. B: At. Mol. Opt. Phys. 48, 065301 (2015).

${ }^{26}$ T. L. Ho, "Spinor Bose condensates in optical traps," Phys. Rev. Lett. 81, 742-745 (1998).

${ }^{27}$ P. G. Kevrekidis, The Discrete Nonlinear Schrödinger Equation: Mathematical Analysis, Numerical Computations, and Physical Perspectives (Springer, Berlin/Heidelberg, 2009).

${ }^{28}$ G. Gligorić, A. Maluckov, Lj. Hadžievski, and B. A. Malomed, "Bright solitons in the one-dimensional discrete Gross-Pitaevskii equation with dipole-dipole interactions," Phys. Rev. A 78, 063615 (2008).

${ }^{29}$ D. A. Zezyulin and V. V. Konotop, "Stationary modes and integrals of motion in nonlinear lattices with a $\mathcal{P} \mathcal{T}$-symmetric linear part", J. Phys. A 46, 415301 (2013).

${ }^{30}$ P. J. Martinez, L. M. Floria, F. Falo, and J. J. Mazo, Intrinsically localized chaos in discrete nonlinear extended systems, Europhys. Lett. 45, 444-449 (1999); V. V. Konotop, D. E. Pelinovsky, and D. A. Zezyulin, Discrete solitons in $\mathcal{P} \mathcal{T}$-symmetric lattices, EPL 100, 56006 (2012).

${ }^{31}$ M. Vakhitov and A. Kolokolov, "Stationary solutions of the wave equation in a medium with nonlinearity saturation," Radiophys. Quantum Electron. 16, 783-789 (1973); L. Bergé, "Wave collapse in physics: Principles and applications to light and plasma waves," Phys. Rep. 303, 259-370 (1998). 